\title{
Approach trajectory and solar position affect host plant attractiveness to the small white butterfly
}

\author{
Adam J. Blake ${ }^{1}$, Samuel Couture ${ }^{1}$, Matthew C. Go ${ }^{1,2}$, Gerhard Gries ${ }^{1}$ \\ ${ }^{1}$ Department of Biological Sciences, Simon Fraser University, Burnaby, British Columbia, Canada \\ ${ }^{2}$ New address: SNA International, supporting the Department of Defense POW/MIA Accounting Agency, Central \\ Identification Laboratory, Joint Base Pearl Harbor-Hickam, Hawaii, USA
}

\begin{abstract}
While it is well documented that insects exploit polarized sky light for navigation, their use of reflected polarized light for object detection has been less well studied. Recently, we have shown that the small white butterfly, Pieris rapae, distinguishes between host and non-host plants based on the degree of linear polarization $(D o L P)$ of light reflected from their leaves. To determine how polarized light cues affect host plant foraging by female $P$. rapae across their entire visual range including the ultraviolet $(300-650 \mathrm{~nm})$, we applied photo polarimetry demonstrating large differences in the DoLP of leaf-reflected light among plant species generally and between host and non-host plants specifically. As polarized light cues are directionally dependent, we also tested, and modelled, the effect of approach trajectory on the polarization of plant-reflected light and the resulting attractiveness to $P$. rapae. Using photo polarimetry measurements of plants under a range of light source and observer positions, we reveal several distinct effects when polarized reflections are examined on a whole-plant basis rather than at the scale of pixels or of entire plant canopies. Most notably from our modeling, certain approach trajectories are optimal for foraging butterflies, or insects generally, to discriminate between plant species on the basis of the DoLP of leaf-reflected light.
\end{abstract}

Keywords: photo polarimetry, polarization vision, axis of polarization, degree of linear polarization, modeling 


\section{List of symbols and abbreviations:}

$\begin{array}{cl}I & \text { intensity } \\ \text { DoLP } & \text { degree of linear polarization } \\ \text { AoP } & \text { axis of polarization } \\ \mathrm{R} & \text { Red (575-700 nm, see Fig. S1c) } \\ \mathrm{G} & \text { Green }(450-625 \mathrm{~nm} \text {, see Fig. S1c) } \\ \mathrm{B} & \text { Blue (400-525 nm, see Fig. S1c) } \\ \mathrm{UV} & \text { Ultraviolet }(325-400 \mathrm{~nm}, \text { see Fig. S1c) } \\ \phi & \text { angle between the azimuth of the observer and the light source (see Fig. 1) } \\ \theta & \text { elevation of light source (see Fig. 1) } \\ \omega & \text { angle between observer and light source with the plant at its vertex (see Fig. 1) } \\ \Psi & \text { 2-dimensional component of } \omega \text { perpendicular to the plane passing through both } \\ & \text { the observer and plant (see Fig. 1) } \\ \zeta & \text { elevation of the observer (see Fig. 1) }\end{array}$


1

2

3

4

5

6

7

8

9

\section{Introduction}

Many insects exploit polarized skylight to aid in navigation (Labhart \& Meyer, 1999) but their use of reflected polarized light for host plant detection and selection has hardly been studied (Heinloth et al., 2018). Recently, the small white butterfly, Pieris rapae (Linnaeus, 1758), which uses cabbage and other crucifers as host plants (Chew \& Renwick, 1995), has been shown to discriminate among host and non-host plants based on the degree of linear polarization $(0-100 \%$, $D o L P$ ) of foliar reflections (Blake et al., 2019). Similar to many other insects (Ilić et al., 2016; Mishra, 2015; Wachmann, 1977), the rhabdom of $P$. rapae photoreceptors is untwisted with uncurved microvilli that are aligned along the rhabdom's length (Blake et al., 2019; Qiu et al., 2002). Rhabdomeric photoreceptors have an inherent dichroism due to the tubular structure of the microvilli (Horváth \& Varjú, 2004). In the ventral compound eye of other insects such as honey bees, Apis mellifera, desert ants, Cataglyphis bicolor, crickets, Gryllus campestris, and cockchafers, Melolontha melolontha, the photoreceptors along with the microvilli composing the rhabdom twist along the photoreceptor's longitudinal axis (Wehner \& Bernard, 1993). This twist serves to disrupt the alignment of microvilli along the rhabdom, preventing preferential absorption of light vibrating in a direction, or with an axis of polarization $\left(0-180^{\circ}, A o P\right)$, parallel to the microvillar orientation, as shown in P. rapae and other insects. Polarization can result in perceived shifts in color and/or intensity as compared to polarization-blind visual systems (Kelber et al., 2001; Kinoshita et al., 2011).

Shiny surfaces like water, glass or plant foliage can polarize light through specular reflection (Foster et al., 2018). These reflections are polarized in a direction so that their AoP is parallel to the surface. The strength of this polarization $(D o L P)$ is dependent on the incident angle, with maximal polarization occurring at the Brewster's angle (approximately $55^{\circ}$ for foliage; Grant et 
24 al., 1993; Johnsen, 2011). The polarization of this light is consequently dependent upon the angle

25 ( $\omega)$ formed between the sun, the reflecting leaf surface, and the observer (i.e., a camera or insect;

26 Fig. 1). This angle is itself dependent upon the solar and observer elevation and azimuth, making

27 these aspects important predictors of foliar polarization (Hegedüs \& Horváth, 2004). As it is only

28 the specular component of the reflection that is polarized, leaf surface characteristics that

29 increase surface roughness and diffuse reflectance, such as pubescence, epicuticular waxes or

30 undulations, also affect the $D o L P$ (Grant et al., 1993). The $D o L P$ can also be altered by reducing

31 diffuse reflectance through pigmentation absorption (Horváth \& Varjú, 1997), resulting in an

32 increased foliar $D o L P$ in the red and blue relative to green.

As $D o L P$ is an important host plant cue, at least for female $P$. rapae (Blake et al., 2019), it

34 would be informative to compare the DoLP and $A o P$ of multiple host and non-host plants. While

35 the polarization of select plant species has previously been examined (Grant et al., 1993), and

36 photo polarimetry has been used to examine plant surfaces (Horváth et al., 2002), photo

37 polarimetry has not yet been used to compare foliar reflected polarized light among different

38 plant species. Moreover, polarization characteristics of foliage in the ultraviolet range (UV, 320-

$39400 \mathrm{~nm}$ ) have been predicted to resemble those in the human-visible range (400-700 $\mathrm{nm})$

40 (Horváth et al., 2002), but this prediction has never been experimentally tested. Therefore, our

41 first objective was to use photo polarimetry to characterize the $D o L P$ and $A o P$ of foliar

42 reflections from host and non-host plants of $P$. rapae and to compare polarization characteristics

43 of foliage in both the UV and human-visible range.

$44 \quad$ Further knowledge gaps pertain to the question as to how interspecific differences in foliar

45 polarization are affected by the position of the observer and the light source. Positional effects

46 have been investigated in relation to single leaves (Hegedüs \& Horváth, 2004; Horváth et al., 
47 2002) but not whole plants. Therefore, our second objective was to use photo polarimetry to

48 measure select plant species under a series of light source and observer positions in order to

49 model how approach trajectory affects foliar $A o P$ and $D o L P$, and thus plant attractiveness, to

50 host-plant-seeking female P. rapae.

\section{Methods}

52 Plant material Within a greenhouse, we grew plants in pots $(12.7 \mathrm{~cm}$ diam $)$, thinning to

53 one plant per pot except for fall rye and oregano. In these species, multiple plants per pot

54 generated a leaf area more comparable to that of the other species examined (Table S1). Plants

55 selected for photography in experiments were 10-20 cm tall with 4-6 fully expanded true leaves

$56(\mathrm{BBCH} 14-16)$.

\section{Polarimetry of Experimental Plants We used photo polarimetry (Foster et al., 2018;}

58 Horváth \& Varjú, 2004) to measure the intensity (I), DoLP and $A o P$ of the selected plants. To

59 obtain these measurements, we used a modified Olympus E-PM1 camera (Olympus, Tokyo,

60 Japan) with expanded sensitivity in the UV (320-400 nm) (Fig S1c; Dr. Klaus Schmitt,

61 Weinheim, Germany, uvir.eu) and an ultra-broadband linear polarizing filter (68-751, Edmund

62 Optics, USA). We narrowed sensitivity to the human-visible range (400-700 nm) and the UV

63 range with a UV/IR filter (Baader Plantarium, Mammendorf, Germany) and a U-filter (Baader

64 Plantarium), respectively. To calculate the $D o L P$ and the $A o P$, we took four images with the

65 polarizing filter positioned at $0^{\circ}, 45^{\circ}, 90^{\circ}$ and $135^{\circ}$.

66 We kept the white-balance, aperture, and other exposure controls constant between

67 exposures, with all images captured in a raw image format. Within the image-analysis software 68 platform Fiji (Schindelin et al., 2012), we used a series of custom-created macros for image 
analysis and measurement (Blake et al., 2020b). We decoded images with DCRAW (Coffin, 2019) as a 16-bit linear bitmap, persevering sensor linearity. We determined color corrections necessary to ensure accurate color representation through photographing a $99 \%$ Spectralon reflectance standard (SRS-99-010, Labsphere, NH, USA) under similar lighting conditions as the experimental plants (Blake et al., 2020b). We aligned all images $\left(0^{\circ}, 45^{\circ}, 90^{\circ}, 135^{\circ}\right)$ from each plant using TurboReg (Thévenaz et al., 1998) and separated the plant in each image from the background (see below). We then calculated Stokes parameters (including I), DoLP, and AoP for each pixel in the red (575-700 nm), green (455-610 nm), blue (410-530 nm) and UV (330-395 $\mathrm{nm}$ ) bands of the electromagnetic spectrum (Fig. S1c) and averaged all pixel values to give a whole-plant mean for both the intensity $(I)$ and the $D o L P$, and a modal value for the $A o P$.

Interspecific comparisons of foliar reflectance (Exp. 1) We photographed plants upright inside a black velvet-lined box lit by a 400 W Hortilux ${ }^{\circledR}$ Blue metal halide lamp (MT400D/BUD/HTL-BLUE, EYE Lighting Int., Mentor, OH, USA) suspended 75-80 cm above the box (Fig. S2). Light was directed onto a plant by a white-cardstock tube $(12.5 \times 21.6 \mathrm{~cm})$, thus minimizing reflections from the box walls. The camera was positioned $75-80 \mathrm{~cm}$ from the plant at approximately the same height as the plant canopy (Fig. S2).

In all exposures $\left(0^{\circ}, 45^{\circ}, 90^{\circ}, 135^{\circ}\right)$ and color bands (UV, blue, green, red), we used a background mask to isolate the plant from the background. We created the background mask using areas above $\sim 2.3 \%$ of the maximum pixel value in the green band. To eliminate possible effects of shading or unequal areas of the plants being directly lit, we limited estimations of $D o L P$ and $A o P$ to areas of the image above $5 \%$ of the maximum pixel value in each color band. We further limited estimates of $A o P$, in this and subsequent experiments, to areas with a $D o L P$ 
91 greater than $15 \%$, as below this DoLP estimates of $A o P$ have little meaning (Horváth \& Varjú,

92 1997).

93 Effect of light source azimuth and elevation on foliar polarization (Exp. 2) To photograph

94 plants in various light source elevation and azimuth combinations (Fig. labce), we used

95 scaffolding to precisely vary the height of the metal halide lamp and a movable platform to keep

96 the camera and plant in orientation. Subtle variations in plant height did result in some variation

97 in light source elevation but these variations and those of related angles were incorporated into

98 the analyses. We positioned a black velvet background behind the plant in each image to enable

99 optimal separation of the plant from the background. We took these measurements using a subset

100 of the species we examined in the previous experiment, selecting plants with shiny leaves

101 (potato, white mustard), matte leaves (cabbage, rutabaga) and fall rye, which holds its leaves in a

102 more vertical orientation. We omitted UV polarimetry in this and the subsequent experiment

103 because plants would shift position due to positive phototropism (Koller, 2000) during the

104 extended time frame needed for several long UV exposures. Omitting UV polarimetry in

105 experiment 2 was further justified given the strong correlation $\left(\mathrm{R}^{2}=75 \%\right)$ between $D o L P$ in the

$106 \mathrm{UV}$ and blue found in experiment 1 (see Results).

107 As the intensity of the black velvet background varied considerably with the position of the

108 metal halide lamp, we could not specify a single intensity threshold to separate the plant from the

109 background as we had in the previous experiment. We therefore used a combination of all three

110 human visual color bands to manually create a background mask. As we wanted to compare the

111 plant in different light source positions, we estimated $D o L P$ from the same subset of pixels

112 specified by the background mask rather than limiting DoLP to areas with a specific intensity, as

113 in the previous experiment. 


$$
\begin{gathered}
R_{s}(\omega)=\left|\frac{\left(n_{1} \cos \frac{\omega}{2}\right)-n_{2} \sqrt{1-\left(\frac{n_{1}}{n_{2}} \sin \frac{\omega}{2}\right)}}{\left(n_{1} \cos \frac{\omega}{2}\right)+n_{2} \sqrt{1-\left(\frac{n_{1}}{n_{2}} \sin \frac{\omega}{2}\right)}}\right|^{2} \\
R_{p}(\omega)=\left|\frac{n_{1} \sqrt{1-\left(\frac{n_{1}}{n_{2}} \sin \frac{\omega}{2}\right)}-\left(n_{2} \cos \frac{\omega}{2}\right)}{n_{1} \sqrt{1-\left(\frac{n_{1}}{n_{2}} \sin \frac{\omega}{2}\right)+\left(n_{2} \cos \frac{\omega}{2}\right)}}\right|^{2} \\
p(\omega)=1-\frac{R_{s}(\omega)}{R_{p}(\omega)}
\end{gathered}
$$


131 across most possible values of $\zeta\left(-15-90^{\circ}\right)$, all possible values of $\phi\left(0-360^{\circ}\right)$, and a selection of $\theta$

132 values $\left(15,45,75^{\circ}\right.$; Blake et al., 2020b). These predictions were limited to the blue color channel

133 as there were insufficient data to fit $A o P$ models for the red and green color bands. Then using

134 the ranges of DoLP and $A o P$ shown to be unattractive to P. rapae (Blake et al., 2019), we

135 modeled approach trajectories that would result in attractive and unattractive polarization

136 characteristics, as well as low $\operatorname{DoLP}(<10 \%$, moderately attractive $)$.

\section{Results}

138 Interspecific comparisons of foliar reflectance (Exp. 1) There were statistically significant

139 differences in both intensity and DoLP among plant species in all color bands (Figs. 2ab, S3ab,

140 S4ab, S5ab; Table S2). In contrast, we found minimal, although sometimes statistically

141 significant, differences in $A o P$ among plant species (Figs. 2c, S3c, S4c, S5c; Table S2).

142 Differences in intensity and DoLP were comparably large in the UV and blue color bands. The

143 comparatively shiny-leaved species had a much higher DoLP than the matt-leaved species, but

144 only in the blue and UV bands (Figs. 2b, S5b), where most $P$. rapae host plants grouped

145 together.

146 Effect of light source azimuth and elevation on foliar polarization (Exp. 2) For all three

147 color bands, there was a strong relationship between $\omega$ and DoLP (Figs. 3, S6, S7; Table S2),

148 with $D o L P$ increasing as $\omega$ approached double the Brewster's angle (53-60). This relationship

149 was less pronounced when the plants were lit more from the side (larger $\psi$ angle). Fall rye with

150 mostly vertical leaf orientation showed a different and weaker relationship between $\omega$ and DoLP

151 (Figs. 3a, S6a, S7a). 
There was an approximately proportional negative relationship between the $\psi$ angle and $A o P$

153 in all color bands (Figs. 4, S8, S9; Table S2). The slope of this relationship was steepest when

154 the light source was behind the observer $(\phi=0)$.

155

156

157

158

159

160

161

162

163

164

165 at a $\phi$ angle of $180^{\circ}$, and a moderately attractive low $D o L P$ area opposite it at a $\phi$ angle of $0^{\circ}$. All

173 other combinations of $\phi$ and $\zeta$ resulted in unattractive DoLPs. Increasing solar elevation $(\theta)$

174 shifted the attractive window downward and the low $D o L P$ area upwards. Increased solar 
175 elevation $(\theta)$ also decreased the size of the attractive window, while increasing the size of the

176 low DoLP area. The $A o P$ had little effect on these windows outside of a small narrowing of the

177 attractive window at low solar elevations $(\theta)$.

\section{Discussion}

179 Our study confirms earlier work demonstrating large differences in DoLP among plant species

180 (Blake et al., 2019; Grant et al., 1993) and refines our understanding of polarized reflections

181 from plant foliage. Unlike previous studies that examined polarized reflections of single leaves,

182 models of leaves, or plant canopies (Grant et al., 1993; Hegedüs \& Horváth, 2004; Horváth et al.,

183 2002; Horváth \& Hegedüs, 2014; Maignan et al., 2009; Raven, 2002; Rondeaux \& Herman,

184 1991; Vanderbilt \& Grant, 1985; Woolley, 1971), we recorded reflections from entire plants

185 thereby revealing several emergent phenomena. Most importantly, our modeling suggests that

186 certain approach trajectories are optimal for foraging insects to discriminate among plant species

187 based on the DoLP of foliar reflections.

188 Our measurements of polarization of foliar reflections are consistent with point-source

189 polarimetry data (Grant et al., 1993), and other photo polarimetry of plant surfaces (Fig. 2,S3-5;

190 Hegedüs and Horváth, 2004; Horváth et al., 2002). As predicted by Horváth et al. (2002), our

191 UV polarimetry data closely resemble those of the human-visible color bands, especially blue,

192 and are consistent with previous measurements in the human-visible range. Similar to previous

193 measurements (Grant et al., 1993; Horváth et al., 2002), glossy, flat and/or dark leaf surfaces

194 have an increased ratio of specular to diffuse reflection and greater DoLP than matte, undulating,

195 and/or bright leaf surfaces. As leaves have low reflectance in the green and red color bands, the

$196 D o L P$ s in the blue and UV color bands expectedly exceeded those in the green and red bands.

197 Moreover, plants with leaves that tend to be held more vertically (e.g., fall rye, onions), and 
198 vertically held leaves. When plants were photographed at or below the level of the leaf canopy 217 (lower $\zeta$ ), the DoLP was reduced (Figs. 5, S10, S11). Similar to the effect of $\psi$, lower $\zeta$ results in 218 a smaller leaf surface reflecting light at the observer, and a larger leaf surface being in shadow or

provide little horizonal surface for specular reflections, had low DoLP values. Despite large differences in leaf shape (simple $v s$ compound) and growth form (all basal leaves $v s$ basal and cauline leaves), there were only small, albeit statistically significant, differences in $A o P$ between plant species (Table S2). These findings in combination with the smaller interspecific differences in intensity relative to $D o L P$ (Table S2), further support the conclusion that foliar DoLP is the visual cue that conveys the most host plant information, especially information about the foliar surface (waxes, pubescence, undulations).

$$
\text { The angle between light source, plant and observer }(\omega) \text { strongly predicted the foliar DoLP }
$$
(Figs. 3, S6, S7) for all color bands, with the strongest polarization at twice the Brewster's angle $\left(53-60^{\circ}\right)$. These data are consistent with both theoretical predictions and other experimental measurements of the effect of viewing angles on DoLP (Horváth et al., 2002; Raven, 2002; Rondeaux \& Herman, 1991; Woolley, 1971). However, the phenomenon of lowering the DoLP with increasing $\Psi$ had not previously been noted and emerges here through whole-plant measurements incorporating multiple leaf surfaces. As the orientation of plant leaves is typically more horizontal than vertical, but not perfectly horizontal, plants lit more from the side than from above (greater $\Psi$ ) have a relatively greater leaf area shadowed by their own leaves. These shadowed areas have a lower $D o L P$, lowering the plants' overall DoLP. Of course, this relationship was absent in fall rye (at least at the growth stage examined) with primarily showing light transmitted through the leaves. Light transmitted through leaves has a low DoLP 
220 due to diffuse scattering by plant tissue, as previously observed in single leaf measurements

221 (Horváth et al., 2002; Vanderbilt \& Grant, 1985).

222 In agreement with prior examinations of foliar polarization, the $A o P$ of all color bands was

223 largely a function of $\psi$ (Figs. 4, S8, S9), with values of $A o P$ moving away from $90^{\circ}$ as the light

224 source was less aligned with the line between the observer and the plant (see Fig. 1a). This

225 relation between $A o P$ and $\psi$ is consistent with previous observations (Horváth et al., 2002;

226 Können, 1985). Although the $A o P$ of a particular plant area did not change much in relation to

227 the light source position, the variety of leaf orientations within a single plant and the curvature of

228 leaf surfaces ensured that at least a portion of the plant showed a specular reflection regardless of

229 the light source's position relative to the plant. Invariably, these areas of specular reflection

230 showed a greater $D o L P$ accounting for much of the observed relationship between $A o P$ and $\psi$.

231 The variety of leaf surface orientations and the resultant $A o P$ s also explains why the relationship

232 between $A o P$ and $\psi$ is shallower than the inversely proportional relationship one could expect.

233 When plants were viewed with the light source directly in front of the observer $\left(\phi=180^{\circ}\right.$; Figs.

234 4, S8, S9), the relationship between $A o P$ and $\psi$ had a reduced slope, a phenomenon being more

235 pronounced when the plant was observed from a higher angle ( $\zeta>0$; Fig. S12). In both cases $(\phi=$

$236180^{\circ}, \zeta>0$ ), this resulted in plants having a higher overall DoLP (Figs. 3, 5), and consequently

237 less leaf surface area (with a $<15 \%$ DoLP) being excluded from estimations of $A o P$. Given that

238 less polarized leaf surface areas showed a weaker relationship between $A o P$ and $\psi$, the overall

239 lower $D o L P$ resulted in a stronger relationship between $A o P$ and $\psi$ as only leaf areas with highest

$240 D o L P$ were above the cutoff. All these effects of $\psi$ on $A o P$ could potentially have biological

241 relevance if a host plant foraging insect were to weigh observations of $A o P$ by their $D o L P$ when

242 determining a plant's overall $A o P$. Nonetheless, in our modeling, these specific effects on $A o P$, 
243 and the effects of $A o P$ in general, on host plant attractiveness to $P$. rapae seem to be subtle in

244 comparison to the effects of DoLP (Figs. 6, S13).

245 Our modeling of the effect of approach trajectory on visual attractiveness of plants to $P$.

246 rapae revealed that $D o L P$ is a much more important determinant of plant attractiveness than $A o P$

247 (Fig. 6). Approach trajectories resulting in $A o P$ unattractive to $P$. rapae were also unattractive

248 due to low DoLP. It follows that the effect of $A o P$ on plant attractiveness can largely be

249 discounted. The key determinant of an attractive DoLP was the azimuth of an approach

250 trajectory relative to the light source $(\phi)$. This was due to its effect on $\psi$, as plants obliquely lit

251 even at the Brewster's angle showed a much lower DoLP. In fact, the only attractive approach

252 trajectories were those where the light source was located behind the target plant. DoLP and

253 attractiveness were also affected by how close the angle between observer, plant and light source

$254(\omega)$ was to twice the Brewster's angle, which is affected by light source elevation $(\theta)$, observer

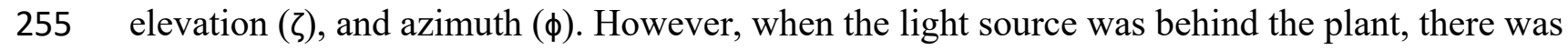

256 always a combination of $\theta$ and $\zeta$ allowing for foliar reflections approaching the Brewster's angle.

257 Although high solar elevations $\left(>75^{\circ}\right)$ - constrained to times near solar noon and limited to

258 latitudes near the equator - are relatively rare, they would require much lower approach angles

259 for accurate assessment of foliar DoLP. It is the key result of our modelling that for most solar

260 positions there is a single optimal approach trajectory that would best enable a foraging insect to

261 assess foliar DoLP. However, this conclusion applies only to settings where foliar reflections are

262 dominated by the specular reflections of sunlight (or another single strong unpolarized light

263 source), as we took measurements indoors and did not incorporate possible effects of polarized

264 skylight (Hegedüs \& Horváth, 2004; Horváth et al., 2002). 
We have every reason to predict that our polarization modeling is applicable to the foliar reflectance of many plant species. However, the data we have obtained with herbaceous flowering plants may not be applicable to graminoids, such as fall rye, or other plants with more vertically held leaves. Moreover, due to the size of trees and large shrubs, foraging insects more often approach them from below (reference), and do not view them in their entirety, complicating the applicability of our modeling. It would therefore be intriguing to model whether

271 approach trajectories have similar effects on polarized light cues that may be used by insect

272 herbivores of trees and shrubs.

273 While this work focused on P. rapae, our DoLP and AoP modeling should be applicable to

274 other polarization-sensitive visual systems. Furthermore, our prediction of a single optimal

275 approach trajectory for the discrimination of $D o L P$ should hold true for other polarization-

276 sensitive insects such as Papilio butterflies (Kelber et al., 2001; Kinoshita et al., 2011), where

277 increased DoLP of foliar reflections would be expected to have a linear effect on attractiveness

278 (Blake et al., 2020a). The approach of butterflies to host plants has not yet been well documented

279 and - accordingly - no stereotyped approach has been noted, as one would anticipate based on

280 our predictions of polarized reflections. Reminiscent of the plunge responses of Notonecta

281 backswimmers (Schwind, 1984), one might expect an approach where the butterflies' trajectory

282 is constrained so that at least a portion of the compound eyes are viewing the plant at or near the

283 Brewster's angle. Alternatively, butterflies might circle plants before landing, thereby shifting

284 their azimuth relative to sun, and entering and exiting the attractive window we identified.

285 Circling plants would also allow for sequential comparison of visual information from the plant

286 surface, aiding in DoLP assessment through differences in color and/or intensity (Horváth \&

287 Varjú, 2004). Mapping the position of butterflies in a 3-dimensional space during approaches to 
288 host plants would give insight into how these insects perceive and use the plants' polarized light 289 cues.

290 In conclusion, using photo polarimetry to examine polarized reflections from entire plants, 291 we show that host and non-host plants of $P$. rapae differ in the DoLP of foliar reflections, with

292 UV measurements closely resembling those of blue. Our photo polarimetry further reveals that

293 there is a single optimal approach trajectory that would enable a foraging insect (or other

294 observers) to best discriminate among these interspecific differences in polarization. This

295 optimal approach trajectory is always in the direction of the light source but its inclination is

296 dependent upon the elevation of the light source $(\theta)$. It would now be intriguing to determine

297 whether the trajectories of polarization-sensitive insects towards host plants match those

298 predicted by our models.

\section{Acknowledgments}

300 This study was supported by an Alexander Graham Bell Canadian Graduate Scholarship to AJB,

301 NSERC Undergraduate Student Research Awards to MCG and SC, and by a NSERC-Industrial

302 Research Chair to GG, with Scotts Canada Ltd. and BASF Canada Ltd. as the industrial partners.

303 Competing Interests The NSERC-Industrial Research Chair to GG was supported by Scotts

304 Canada Ltd. and BASF Canada Ltd. as industrial partners.

305 Data Accessibility Data are available from Mendeley Data:

306 http://doi.org/10.17632/5bh5mhmvrk.1 (Blake et al., 2020b).

307 Author Contributions AJB, SC, MCG performed the polarimetry; AJB designed experiments;

308 AJB performed modeling and analyses; GG supervised the project; AJB wrote the first draft of 309 the paper and GG provided comments. 
310

311

312

313

314

315

316

317

318

319

320

321

322

323

324

325

326

327

328

329

330

331

\section{References}

Bates, D., Mächler, M., Bolker, B., \& Walker, S. (2015). Fitting linear mixed-effects models using Ime4. Journal of Statistical Software, 67(1), 1-48. https://doi.org/10.18637/jss.v067.i01

Blake, Adam J., Hahn, G. S., Grey, H., Kwok, S. A., McIntosh, D., \& Gries, G. (2020a). Polarized light sensitivity in Pieris rapae is dependent on both color and intensity. Journal of Experimental Biology, 223(13), jeb220350. https://doi.org/10.1242/jeb.220350

Blake, Adam J., Couture, S., Go, M. C., Gries, G. (2020b), Polarimetry data, ImageJ/FIJI macros, R modeling code, and other data from: Approach trajectory and solar position affect host plant attractiveness to the small white butterfly, Mendeley Data, V1, http://doi.org/10.17632/5bh5mhmvrk.1

Blake, Adam J., Pirih, P., Qiu, X., Arikawa, K., \& Gries, G. (2019). Compound eyes of the small white butterfly Pieris rapae have three distinct classes of red photoreceptors. Journal of Comparative Physiology A, 205(4), 553-565. https://doi.org/10.1007/s00359-019-013308

Blake, Adam James, Go, M. C., Hahn, G. S., Grey, H., Couture, S., \& Gries, G. (2019). Polarization of foliar reflectance: novel host plant cue for insect herbivores. Proceedings of the Royal Society B: Biological Sciences, 286, 20192198. https://doi.org/10.1098/rspb.2019.2198

Chew, F. S., \& Renwick, J. A. A. (1995). Host plant choice in Pieris butterflies. In Chemical ecology of insects 2 (Vol. 1-Chapter 6, pp. 214-238). Springer. https://doi.org/10.1007/978-1-4615-1765-8_6 
Coffin, D. (2019, December 23). Decoding raw digital photos in Linux. https://www.dechifro.org/dcraw/

Foster, J. J., Temple, S. E., How, M. J., Daly, I. M., Sharkey, C. R., Wilby, D., \& Roberts, N. W. (2018). Polarisation vision: overcoming challenges of working with a property of light we barely see. The Science of Nature, 105, 27. https://doi.org/10.1007/s00114-018-1551-

Grant, L., Daughtry, C. S. T., \& Vanderbilt, V. C. (1993). Polarized and specular reflectance variation with leaf surface features. Physiologia Plantarum, 88(1), 1-9. https://doi.org/10.1111/j.1399-3054.1993.tb01753.x

Heinloth, T., Uhlhorn, J., \& Wernet, M. F. (2018). Insect responses to linearly polarized

Hegedüs, R., \& Horváth, G. (2004). Polarizational colours could help polarization-dependent colour vision systems to discriminate between shiny and matt surfaces, but cannot unambiguously code surface orientation. Vision Research, 44(20), 2337-2348.

Horváth, G, Gál, J., Labhart, T., \& Wehner, R. (2002). Does reflection polarization by plants reflections: orphan behaviors without neural circuits. Frontiers in Cellular Neuroscience, influence colour perception in insects? Polarimetric measurements applied to a polarization-sensitive model retina of Papilio butterflies. Journal of Experimental 12, 50. https://doi.org/10.3389/fncel.2018.00050 https://doi.org/10.1016/j.visres.2004.05.004 Biology, 205(21), 3281-3298. https://jeb.biologists.org/content/205/21/3281

Horváth, G, \& Varjú, D. (1997). Polarization pattern of freshwater habitats recorded by video polarimetry in red, green and blue spectral ranges and its relevance for water detection by 
aquatic insects. Journal of Experimental Biology, 200(7), 1155-1163.

355 https://jeb.biologists.org/content/200/7/1155

Horváth, Gábor, \& Hegedüs, R. (2014). Polarization Characteristics of Forest Canopies with Biological Implications. In Gábor Horváth (Ed.), Polarized Light and Polarization Vision in Animal Sciences (pp. 345-365). Springer Berlin Heidelberg. https://doi.org/10.1007/978-3-642-54718-8_17

Horváth, Gábor, \& Varjú, D. (2004). Polarized Light in Animal Vision: Polarization Patterns in Nature. Springer.

Ilić, M., Pirih, P., \& Belušič, G. (2016). Four photoreceptor classes in the open rhabdom eye of the red palm weevil, Rynchophorus ferrugineus Olivier. Journal of Comparative

Johnsen, S. (2011). The Optics of Life. Princeton University Press. http://lib.myilibrary.com.proxy.lib.sfu.ca/Open.aspx?id=343974

367 Kelber, A., Thunell, C., \& Arikawa, K. (2001). Polarisation-dependent colour vision in Papilio. Journal of Experimental Biology, 204(14), 2469-2480. https://jeb-biologistsorg.proxy.lib.sfu.ca/content/204/14/2469.short

Kinoshita, M., Yamazato, K., \& Arikawa, K. (2011). Polarization-based brightness discrimination in the foraging butterfly, Papilio xuthus. Philosophical Transactions of the Royal Society B: Biological Sciences, 366(1565), 688-696. https://doi.org/10.1098/rstb.2010.0200

Koller, D. (2000). Plants in search of sunlight. In Advances in Botanical Research (Vol. 33, pp. 35-131). Elsevier. https://doi.org/10.1016/S0065-2296(00)33041-5 
Können, G. P. (1985). Polarized Light in Nature. Cambridge University Press. https://books.google.ca/books?id=OBY9AAAAIAAJ\&lpg=PP1\&dq=Polarized\%20Light $\% 20$ in $\% 20$ Nature $\% 201985$

Labhart, T., \& Meyer, E. P. (1999). Detectors for polarized skylight in insects: a survey of ommatidial specializations in the dorsal rim area of the compound eye. Microscopy Research \& Technique, 47(6), 368-379. https://doi.org/10.1002/(SICI)10970029(19991215)47:6<368::AID-JEMT2>3.0.CO;2-Q

Maignan, F., Bréon, F.-M., Fédèle, E., \& Bouvier, M. (2009). Polarized reflectances of natural surfaces: Spaceborne measurements and analytical modeling. Remote Sensing of Environment, 113(12), 2642-2650. https://doi.org/10.1016/j.rse.2009.07.022

Mishra, M. (2015). An eye ultrastructure investigation of a plant pest Acyrthosiphon pisum (Harris) (Insecta: Hemiptera: Aphididae). Open Access Insect Physiology, 5, 41-46. https://doi.org/10.2147/OAIP.S84633

Qiu, X., Vanhoutte, K., Stavenga, D. G., \& Arikawa, K. (2002). Ommatidial heterogeneity in the compound eye of the male small white butterfly, Pieris rapae crucivora. Cell and Tissue Research, 307(3), 371-379. https://doi.org/10.1007/s00441-002-0517-z

Raven, P. N. (2002). Polarized directional reflectance from laurel and mullein leaves. Optical Engineering, 41(5), 1002-1011. https://doi.org/10.1117/1.1467668

Rondeaux, G., \& Herman, M. (1991). Polarization of light reflected by crop canopies. Remote Sensing of Environment, 38(1), 63-75. https://doi.org/10.1016/0034-4257(91)90072-E

Schindelin, J., Arganda-Carreras, I., Frise, E., Kaynig, V., Longair, M., Pietzsch, T., Preibisch, S., Rueden, C., Saalfeld, S., Schmid, B., Tinevez, J.-Y., White, D. J., Hartenstein, V., Eliceiri, K., Tomancak, P., \& Cardona, A. (2012). Fiji: an open-source platform for 
biological-image analysis. Nature Methods, 9(7), 676-682. https://doi.org/10.1038/nmeth.2019

401 Schwind, R. (1984). The plunge reaction of the backswimmer Notonecta glauca. Journal of Comparative Physiology A, 155(3), 319-321. https://doi.org/10.1007/BF00610585

Thévenaz, P., Ruttimann, U. E., \& Unser, M. (1998). A pyramid approach to subpixel registration based on intensity. IEEE Transactions on Image Processing : A Publication on Geoscience and Remote Sensing, GE-23(5), 722-730. https://doi.org/10.1109/TGRS.1985.289390

Wachmann, E. (1977). Vergleichende Analyse der feinstrukturellen Organisation offener Rhabdome in den Augen der Cucujiformia (lnsecta, Coleoptera), unter besonderer Berücksichtigung der Chrysomelidae. Zoomorphologie, 88(2), 95-131.

413 Wehner, R., \& Bernard, G. D. (1993). Photoreceptor twist: a solution to the false-color problem. Proceedings of the National Academy of Sciences, 90(9), 4132-4135. https://doi.org/10.1073/pnas.90.9.4132

416 Woolley, J. T. (1971). Reflectance and transmittance of light by leaves. Plant Physiology, 47(5), 656-662. https://doi.org/10.1104/pp.47.5.656 

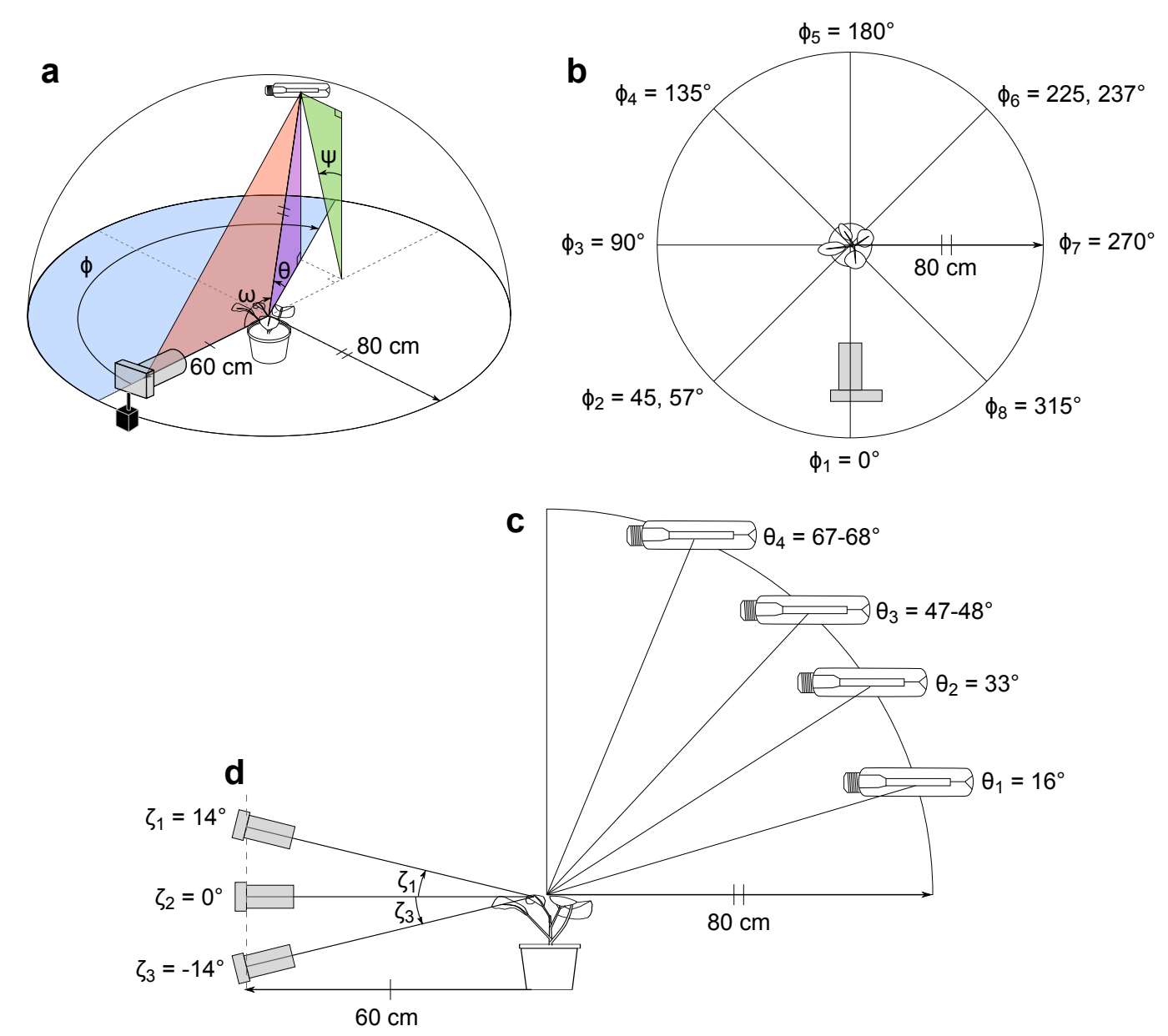

\begin{tabular}{|c|c|c|c|c|c|c|c|c|c|c|c|c|c|c|c|}
\hline \multirow{3}{*}{$\begin{array}{c}\mathrm{e} \\
\text { angles }\end{array}$} & \multicolumn{15}{|c|}{$\phi$ angles } \\
\hline & \multicolumn{5}{|c|}{${ }^{1} \exp .2,0-180^{\circ}$} & \multicolumn{5}{|c|}{${ }^{1}$ exp. $2,180-360^{\circ}$} & \multicolumn{5}{|c|}{ exp. 3} \\
\hline & $0^{\circ}$ & $45^{\circ}$ & $90^{\circ}$ & $135^{\circ}$ & & & $237^{\circ}$ & $270^{\circ}$ & $315^{\circ}$ & $0^{\circ}$ & & & & & \\
\hline $33^{\circ}$ & $0^{\circ}$ & $45^{\circ}$ & $90^{\circ}$ & $135^{\circ}$ & $180^{\circ}$ & & & & & & $0^{\circ}$ & $45^{\circ}$ & $90^{\circ}$ & $135^{\circ}$ & \\
\hline $47-48^{\circ}$ & $0^{\circ}$ & $45^{\circ}$ & $90^{\circ}$ & $135^{\circ}$ & $180^{\circ}$ & $180^{\circ}$ & $225^{\circ}$ & $270^{\circ}$ & $315^{\circ}$ & $0^{\circ}$ & $0^{\circ}$ & $45^{\circ}$ & $90^{\circ}$ & $135^{\circ}$ & $180^{\circ}$ \\
\hline $67-68^{\circ}$ & ${ }^{2} 0^{\circ}$ & $57^{\circ}$ & $90^{\circ}$ & $135^{\circ}$ & ${ }^{2} 180^{\circ}$ & ${ }^{2} 180^{\circ}$ & $225^{\circ}$ & $270^{\circ}$ & & ${ }^{2} 0^{\circ}$ & & & & & \\
\hline
\end{tabular}

Figure 1. a Diagram showing the relative position of the camera, experimental plant and light source as well as the angles between them. The differences in azimuth between the camera and the light source $(\phi)$, and the elevation of the light source $(\theta)$, were manipulated to produce a range of values in the angles $\omega \& \psi$. — b The range of values for the angle $\phi$. — c The range of values for the angle $\theta$. - $\mathbf{d}$ The range of values of camera inclination ( $\zeta)$. - $\mathbf{e}$ The degree of linear polarization $(D o L P)$ and axis of polarization $(A o P)$ were measured using photo polarimetry at each combination of $\phi$ and $\theta$ angles listed in the table for experiments 2 and 3 . Due to restrictions of the scaffolding for mounting the metal halide lamp, certain combinations of $\phi$ and $\theta$ were impractical for polarimetry (shown in dark grey). For similar reasons, measurements in experiment 3 were limited to a subset of $\theta$ angles, but for each of the $\phi$ and $\theta$ combinations listed in the table, measurements were taken at each $\zeta$ value. ${ }^{1}$ In experiment 2 , plants were either photographed at a $\phi$ between $0-180^{\circ}$ or $180-360^{\circ} .{ }^{2}$ Due to low $D o L P$, these combinations were excluded from $A o P$ analyses. 

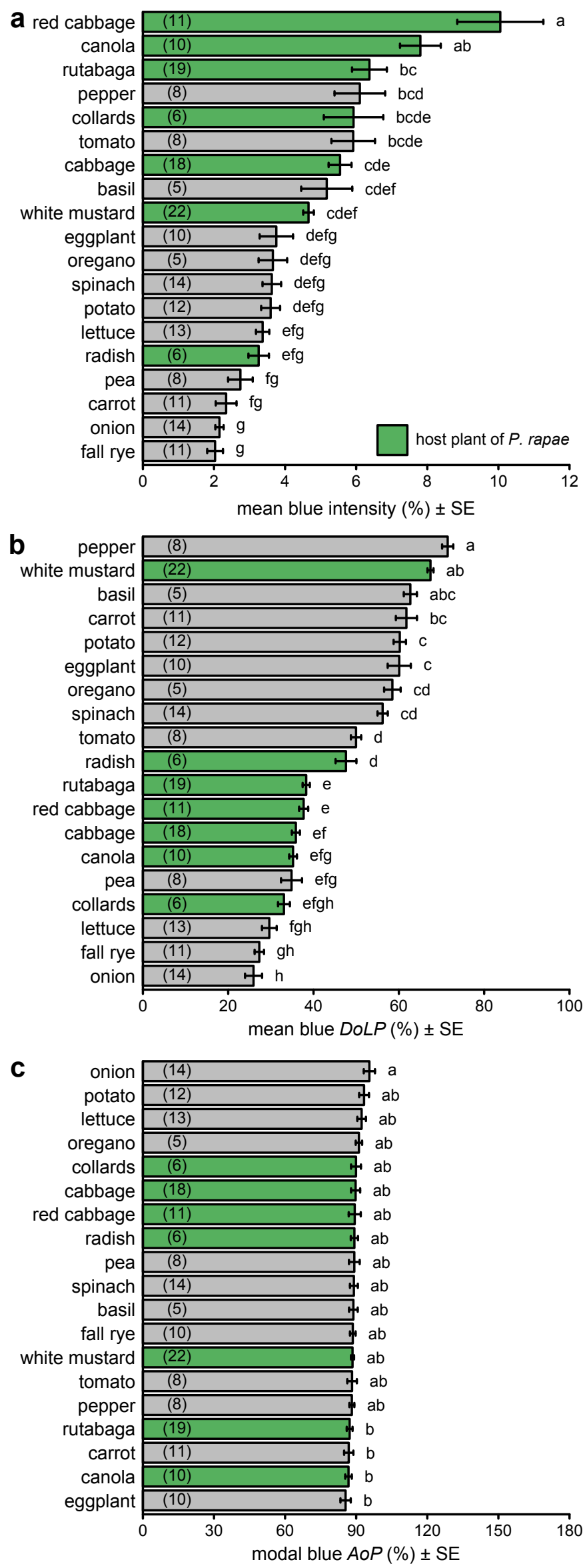

Figure 2. Comparison of intensity (a), degree of linear polarization $(D o L P)(\mathbf{b})$, and axis of polarization $(A o P)(\mathbf{c})$ among host plants (green bars) and non-host plants (grey bars) of Pieris rapae. These measurements used the blue color band, whereas measurements with other color bands are presented in Figs. S3-5. Bars show mean or modal values with the number of plants measured noted in parentheses in each bar. In each subpanel, bars with different letters differ statistically $(p<0.05)$, as determined by a posthoc Tukey test. Data in subpanels $\mathbf{b}$ and $\mathbf{c}$ were previously reported (Blake et al. 2019). 


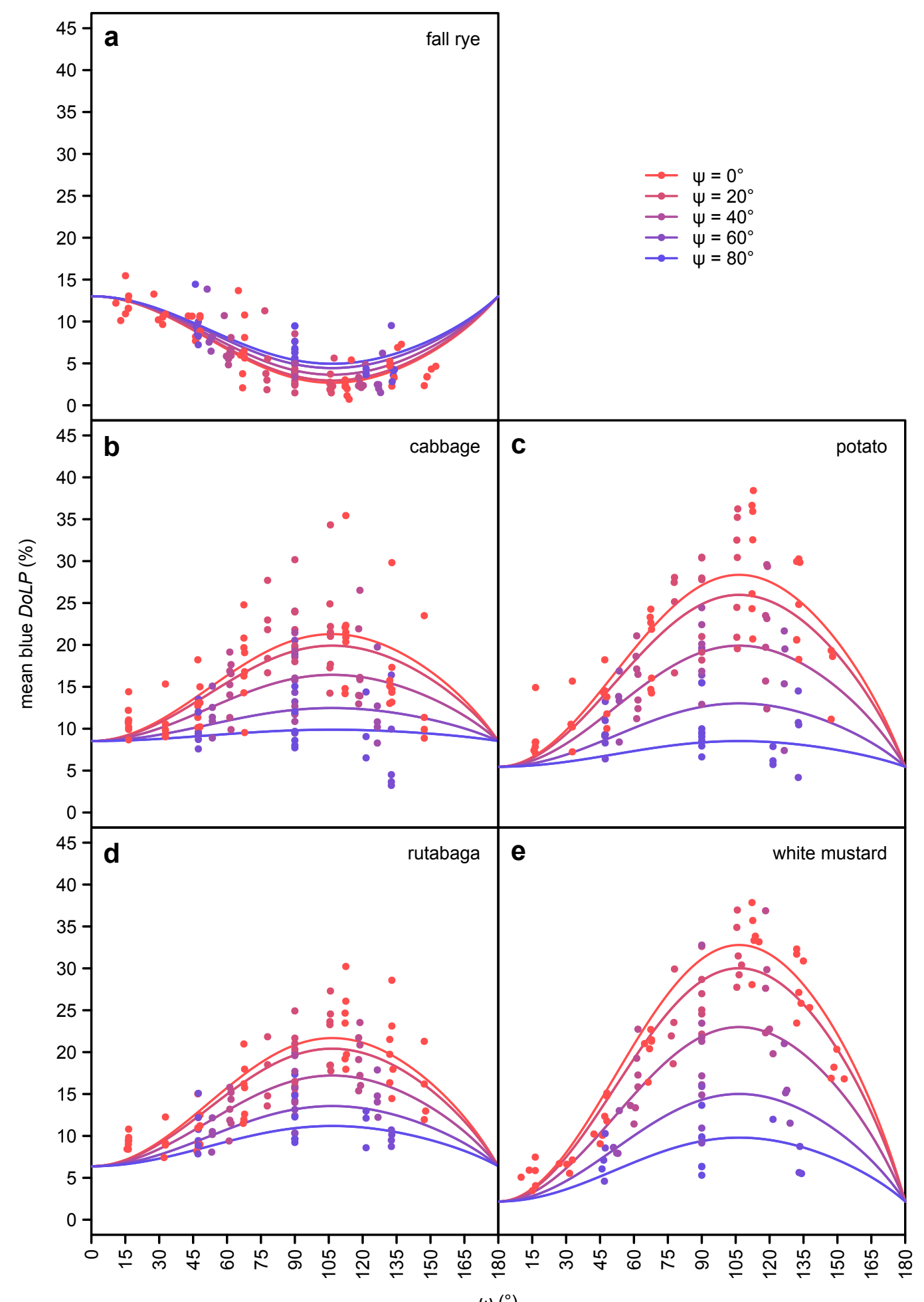

Figure 3. The effect of $\omega$ (angle between observer and light source with the plant at its vertex; see Fig. 1) and $\psi$ (2-dimensional component of $\omega$ perpendicular to the plane passing through both the observer and the plant; see Fig. 1) on the mean degree of linear polarization (DoLP) of the blue color band, as measured in five select plant species using photo polarimetry. Data with other color bands are presented in Figs. S6-7 and show a similar relationship. Cabbage, rutabaga and white mustard are host plants of Pieris rapae. 


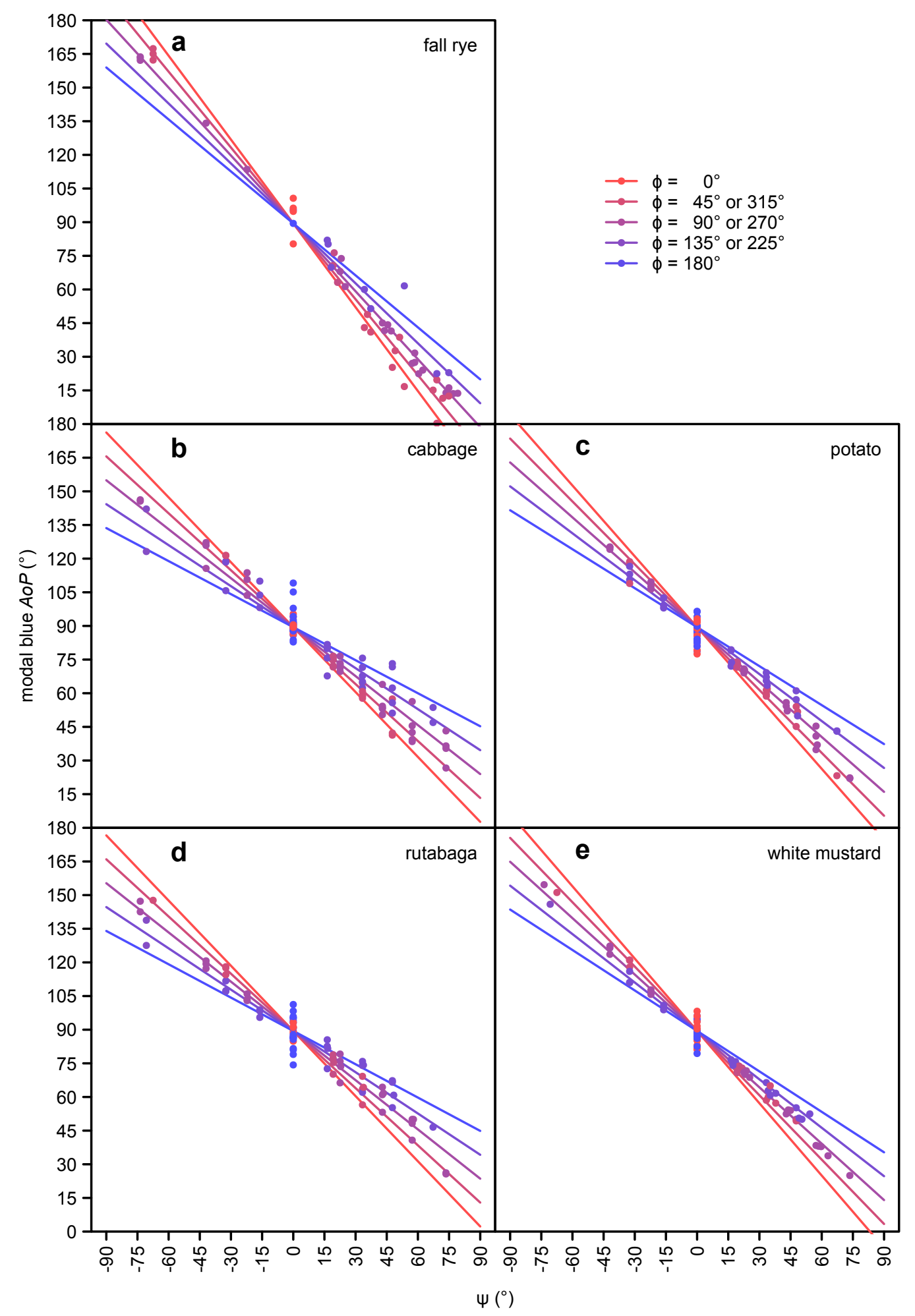

Figure 4. The effect of $\psi$ (2-dimensional component of $\omega$ perpendicular to the plane passing through both the observer and the plant; see Fig. 1) and $\phi$ (angle between the azimuth of the observer and the light source; see Fig. 1) on the modal axis of polarization $(A o P)$ of the blue color band, as measured in five select plant species using photo polarimetry. Data of other color bands are presented in Figs. S8-9 and show a similar relationship. Cabbage, rutabaga and white mustard are host plants of Pieris rapae. 


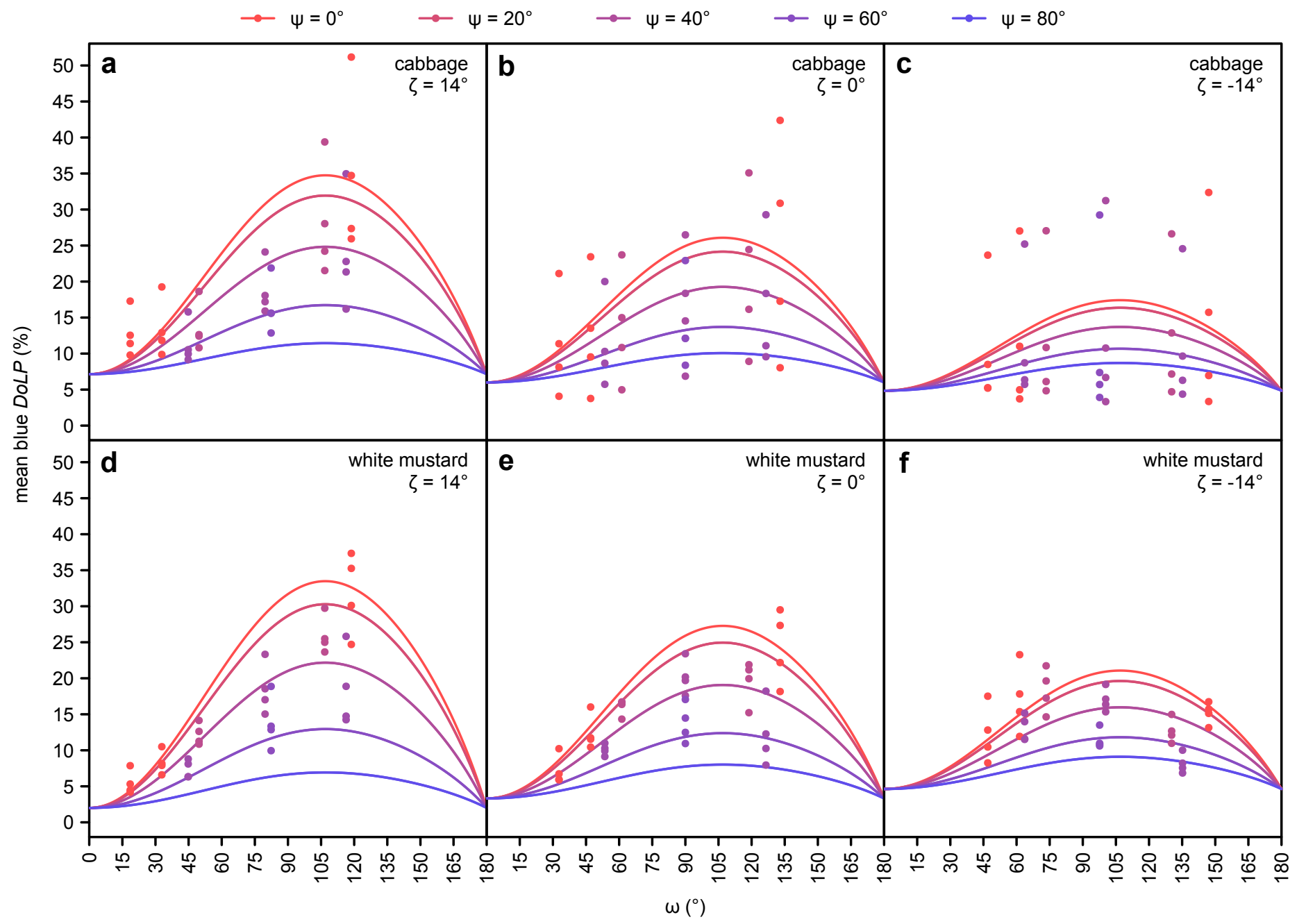

Figure 5. The additional effect of observer elevation ( $\zeta$; see Fig. 1$)$ on the mean degree of linear polarization $(D o L P)$ of the blue color band, as measured in cabbage and white mustard (host plants of Pieris rapae) using photo polarimetry. Data with other color bands are presented in Figs. S10-11 and show a similar relationship. 


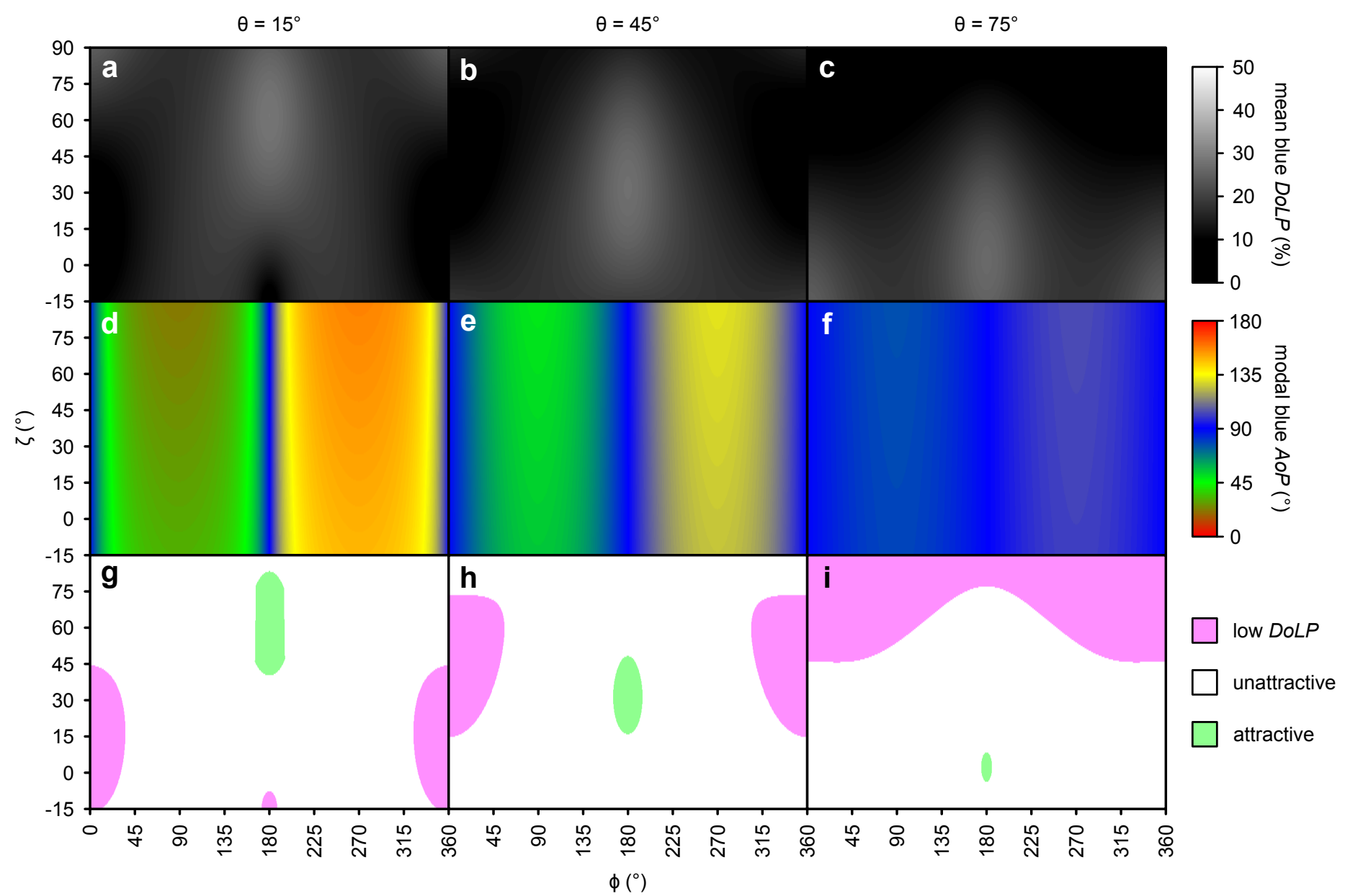

Figure 6. Effects of approach direction (angle between the azimuth of the observer and the light source $(\phi$; see Fig. 1) and elevation of the observer ( $\zeta$; see Fig. 1) on the mean degree of linear polarization $(D o L P)(\mathbf{a}-\mathbf{c})$ and the modal axis of polarization $(A o P)(\mathbf{d - f})$ of the blue color band of cabbage plants (host of Pieris rapae). Attractiveness of resulting polarization characteristics to P. rapae (g-i), based on a previous behavioral study (Blake et al. 2019). Approach trajectories resulting in attractive characteristics $\left(D o L P=26-36 \%\right.$ and $A o P=0-38,53-128$ or $\left.143-180^{\circ}\right)$ and unattractive characteristics $\left(D o L P=10-26 \%\right.$ or $\left.A o P=38-53^{\circ}, 128-143^{\circ}\right)$ are shown in green and white, respectively, with pink indicating trajectories resulting in a moderately-attractive low $\operatorname{DoLP}(<10 \%)$. Higher $\operatorname{DoLP}(36-60 \%)$ would also be unattractive but were not predicted by these models. These effects changed with light source elevation $(\theta$; see Fig. 1$)$ which is shown at $15^{\circ}(\mathbf{a}, \mathbf{d}, \mathbf{g}), 45^{\circ}(\mathbf{b}, \mathbf{e , ~ h})$ and $75^{\circ}(\mathbf{c}, \mathbf{f}, \mathbf{i})$. Analogous data were obtained with white mustard (Fig. S13), another host plant of Pieris rapae. 
Table S1. Variety and taxonomic information of select host plants (green) and non-host plants (black) of Pieris rapae.

\begin{tabular}{|c|c|c|c|}
\hline common name & latin name & variety & family \\
\hline onion & Allium cepa $\mathrm{L}$. & Early Yellow Globe & Amaryllidaceae \\
\hline fall rye & Secale cereale $\mathrm{L}$. & - & Poaceae \\
\hline pea & Pisum sativum $\mathrm{L}$. & Green Arrow & Fabaceae \\
\hline radish & Raphanus raphanistrum L. sativus & Cherry Belle & Brassicaceae \\
\hline rutabaga & Brassica napus L. var. napobrassica & Laurentian Swede & Brassicaceae \\
\hline canola & Brassica napus L. napus f. annua & Q2 & Brassicaceae \\
\hline collards & Brassica oleracea L. var. acephala & Vates & Brassicaceae \\
\hline cabbage & Brassica oleracea L. var. capitata f. alba & Early Jersey Wakefield & Brassicaceae \\
\hline red cabbage & Brassica oleracea L. var. capitata f. rubra & Red Acre & Brassicaceae \\
\hline white mustard & Sinapis alba L. & AC Pennant & Brassicaceae \\
\hline spinach & Spinacia oleracea L. & King of Denmark & Amaranthaceae \\
\hline lettuce & Lactuca sativa L. & Grand Rapids & Asteraceae \\
\hline carrot & Daucus carota L. sativus & Nantes Coreless & Apiaceae \\
\hline basil & Ocimum basilicum L. & Genovese & Lamiaceae \\
\hline oregano & Origanum vulgare L. & - & Lamiaceae \\
\hline eggplant & Solanum melongena L. & Black Beauty & Solanaceae \\
\hline pepper & Capsicum annuum L. & Keystone Resistant & Solanaceae \\
\hline tomato & Solanum lycopersicum L. & Celebrity & Solanaceae \\
\hline potato & Solanum tuberosum L. & Russett Burbank & Solanaceae \\
\hline
\end{tabular}


Table S2. Model statements, test statistics, and p-values for statistical models of photo polarimetry determined measurements of intensity $(I)$, degree of linear polarization $(D o L P)$, and axis of polarization $(A o P)$ for the red $(\mathrm{R})$, green $(\mathrm{G})$, blue $(\mathrm{B})$, ultraviolet (UV, Exp. 1 only) color bands in experiments 1-3. The angles $(\phi, \theta, \omega, \psi, \zeta)$ in the model statements are described in Fig. 1. The $p(\omega)$ relationship is defined in equations 1-3. The fixed effect of different plant species in the model is represented by species, whereas the random effect of individual plants was fit as an intercept and is represented by ( 1 | plant). The full $\mathrm{R}$ code used for statistical analysis is presented in an associated Dryad dataset (Blake et al., 2020b).

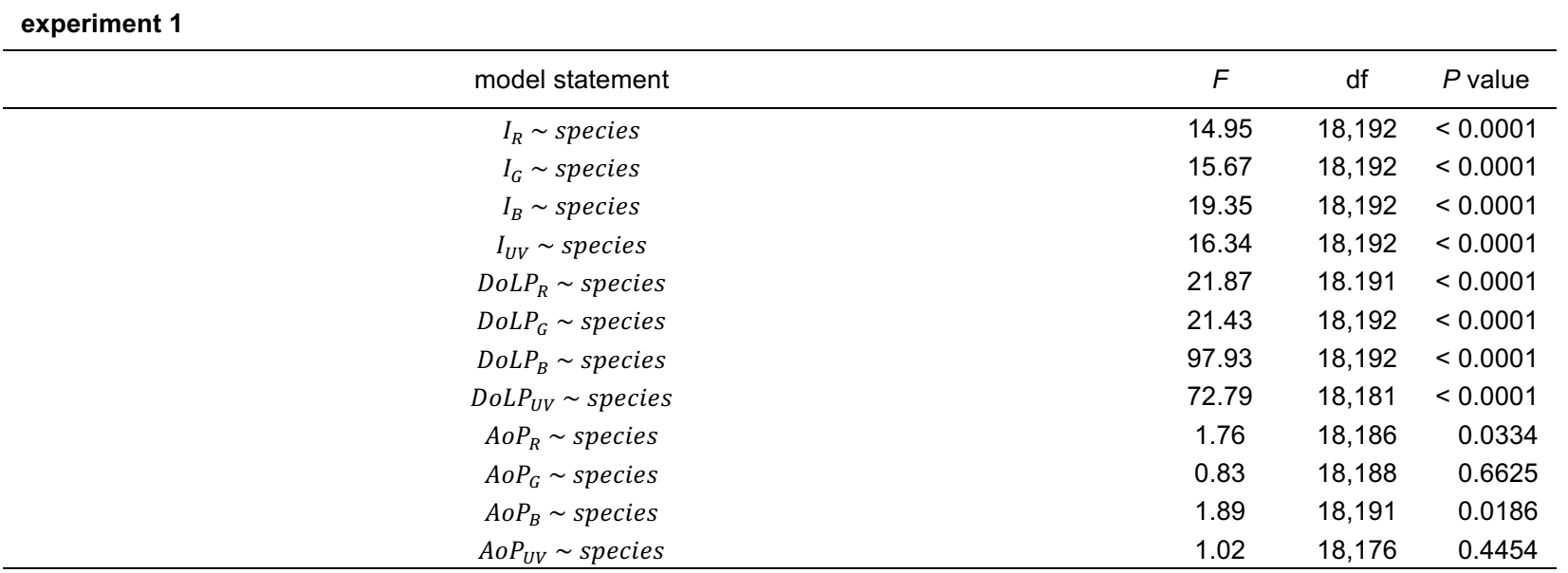

experiment 2

\begin{tabular}{|c|c|c|c|}
\hline model statement & $x^{2}$ & $\mathrm{df}$ & $P$ value \\
\hline $\operatorname{DoLP}_{R} \sim p(\omega)+$ species $+p(\omega):$ species $+\mathrm{p}(\omega): \cos \psi^{2}+p(\omega):$ species $: \cos \psi^{2}+(1 \mid$ plant $)$ & 1049 & 14 & $<0.0001$ \\
\hline DoLP $P_{G} \sim p(\omega)+$ species $+p(\omega):$ species $+\mathrm{p}(\omega): \cos \psi^{2}+p(\omega):$ species $: \cos \psi^{2}+(1 \mid$ plant $)$ & 1059 & 14 & $<0.0001$ \\
\hline DoLP $P_{B} \sim p(\omega)+$ species $+p(\omega):$ species $+p(\omega): \cos \psi^{2}+p(\omega):$ species $: \cos \psi^{2}+(1 \mid$ plant $)$ & 1073 & 14 & $<0.0001$ \\
\hline$A o P_{R} \sim \psi+\psi: \phi+\psi:$ species $+(1 \mid$ plant $)$ & 912 & 5 & $<0.0001$ \\
\hline$A o P_{G} \sim \psi+\psi: \phi+\psi:$ species $+(1 \mid$ plant $)$ & 801 & 5 & $<0.0001$ \\
\hline$A o P_{B} \sim \psi+\psi: \phi+\psi:$ species $+(1 \mid$ plant $)$ & 1267 & 6 & $<0.0001$ \\
\hline
\end{tabular}

experiment 3

\begin{tabular}{|c|c|c|c|}
\hline model statement & $x^{2}$ & $\mathrm{df}$ & $P$ value \\
\hline $\begin{array}{c}\text { DoLP } P_{R} \sim p(\omega)+\text { species }+ \text { atan }(16 \cdot \zeta)+p(\omega) * \text { species }+p(\omega): \cos \psi^{2}+\operatorname{species}: \operatorname{atan}(16 \cdot \zeta) \\
+p(\omega): \text { species }: \cos \psi^{2}+p(\omega): \operatorname{atan}(16 \cdot \zeta): \cos \psi^{2}+(1 \mid \text { plant })\end{array}$ & 342 & 8 & $<0.0001$ \\
\hline $\begin{array}{c}\text { DoLP } P_{G} \sim p(\omega)+\text { species }+ \text { atan }(16 \cdot \zeta)+p(\omega) * \text { species }+p(\omega): \cos \psi^{2}+\text { species }: \text { atan }(16 \cdot \zeta) \\
+p(\omega): \text { species }: \cos \psi^{2}+p(\omega): \text { atan }(16 \cdot \zeta): \cos \psi^{2}+(1 \mid \text { plant })\end{array}$ & 386 & 8 & $<0.0001$ \\
\hline $\begin{array}{c}\text { DoLP } P_{B} \sim p(\omega)+\text { species }+ \text { atan }(16 \cdot \zeta)+p(\omega) * \text { species }+p(\omega): \cos \psi^{2}+\text { species }: \text { atan }(16 \cdot \zeta) \\
\quad+p(\omega): \text { species }: \cos \psi^{2}+p(\omega): \text { atan }(16 \cdot \zeta): \cos \psi^{2}+(1 \mid \text { plant })\end{array}$ & 284 & 8 & $<0.0001$ \\
\hline $\begin{array}{c}\text { AoP } P_{R} \sim \psi+\psi: \phi+\psi: \text { atan }(16 \cdot \zeta)+\psi: \text { species }+\psi: \phi: \operatorname{atan}(16 \cdot \zeta) \\
+\psi: \text { species }: \operatorname{atan}(16 \cdot \zeta)+(1 \mid \text { plant })\end{array}$ & 419 & 6 & $<0.0001$ \\
\hline
\end{tabular}



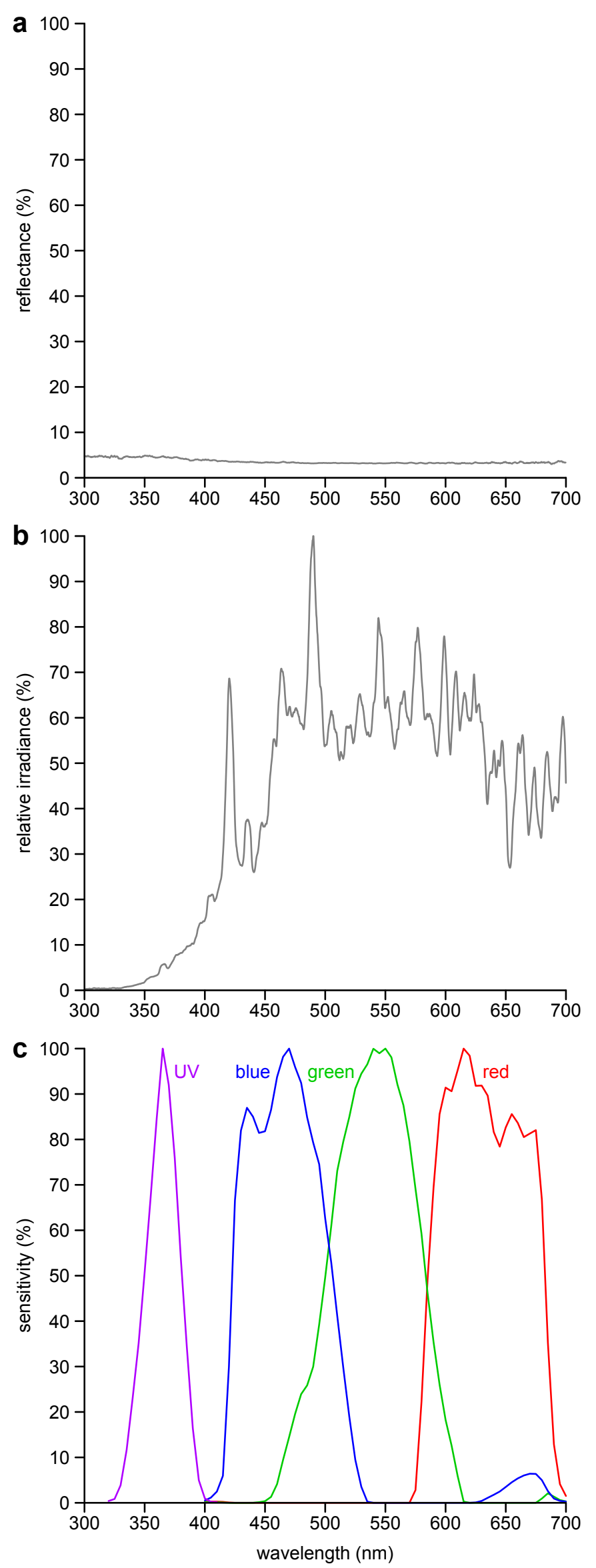

Figure S1. Spectra of background, illumination sources, and camera sensitivity. a, Reflection spectrum of the black velvet background. b, Relative irradiance of the metal halide lamp. $\mathbf{c}$, Spectral sensitivity of the modified Olympus EPM1 camera in the ultraviolet (UV), blue, green and red bands of the electromagnetic spectrum. Reflectance spectra were measured with a JAZ spectrometer (Ocean Optics Inc., Dunedin, FL, USA) calibrated with a $99 \%$ Spectralon reflectance standard (SRS-99-010, Labsphere, $\mathrm{NH}$, USA). Irradiance spectra were measured with a calibrated HR-4000 spectrophotometer (Ocean Optics Inc.). Isoquantal monochromatic light for spectral sensitivity determination was generated with the same HR-4000 spectrophotometer and a scanning monochromator (MonoScan 2000, Mikropak GmbH, Ostfildern, Germany). 

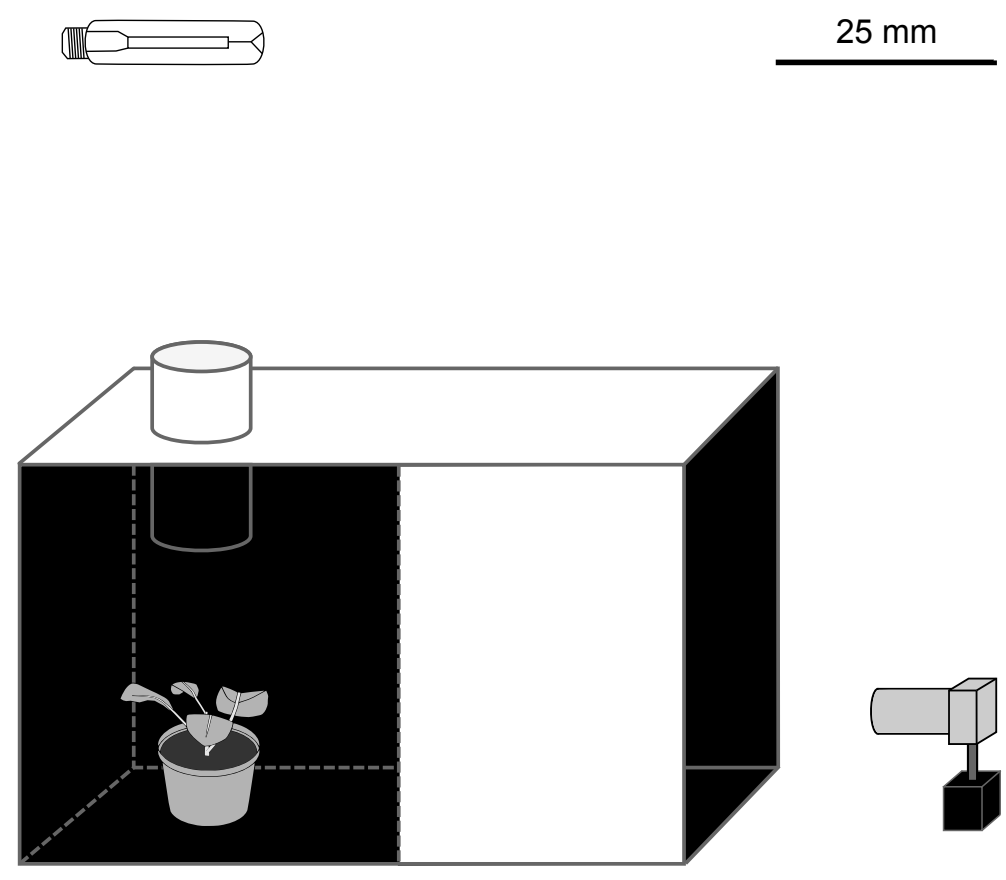

Figure S2. Design for photo polarimetry deployed to characterize the intensity, degree, and axis of linear polarization of various host and non-host plants of Pieris rapae in the red, green, blue, and ultraviolet color bands. The camera was positioned so that its optical axis was level with the plant canopy. The plant was positioned underneath the spotlight to avoid illumination of box walls. The angle between the camera and the light source was approximately $90^{\circ}$. 

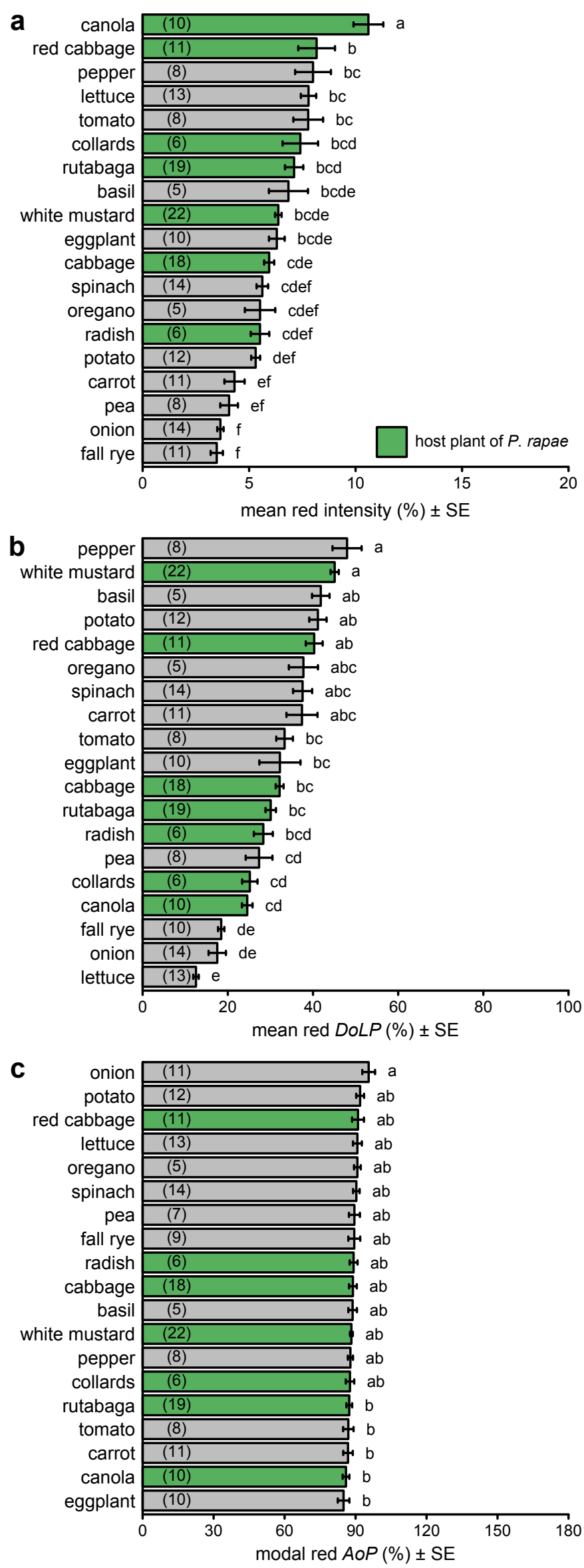

Figure S3. Comparison of intensity (a), degree of linear polarization $(D o L P)(\mathbf{b})$, and axis of polarization $(A o P)(\mathbf{c})$ among host plants (green bars) and non-host plants (grey bars) of Pieris rapae. These measurements used the red color band. Bars show mean or modal values with number of plants measured noted in parentheses in each bar. Bars with different letters differ statistically $(p<0.05)$, as determined by a posthoc Tukey test. 
a

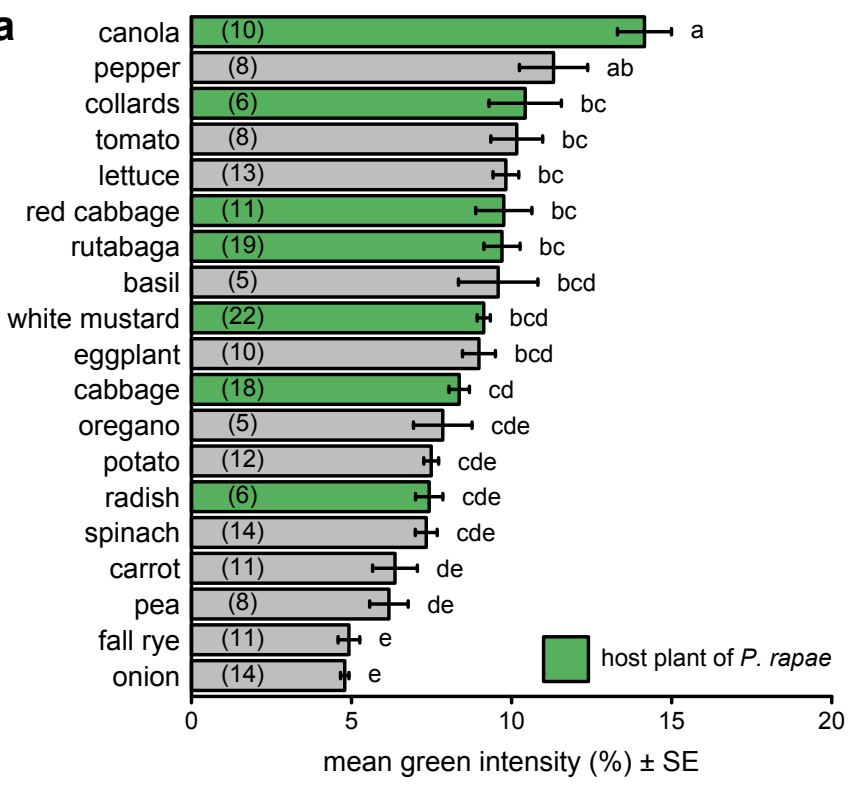

b red cabbage $(11) \quad-\quad$ a

pepper (8)

white mustard

basil (5) $\quad$ abc

tomato

potato $(12) \quad$ A $\quad$ bc

eggplant $(10)$

spinach $(14) \quad 7 \mathrm{bc}$

oregano (5) $\quad$ bcd

carrot (11)

rutabaga

cabbage $(18) \quad c d$

canola (10) \& cde

radish (6) cde

collards (6)

pea $(8) \quad$ t def

fall rye (11) def

onion (14) ef

lettuce (13) $\mathrm{f}$

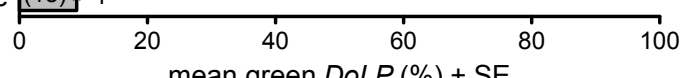

mean green $\operatorname{DoLP}(\%) \pm \mathrm{SE}$

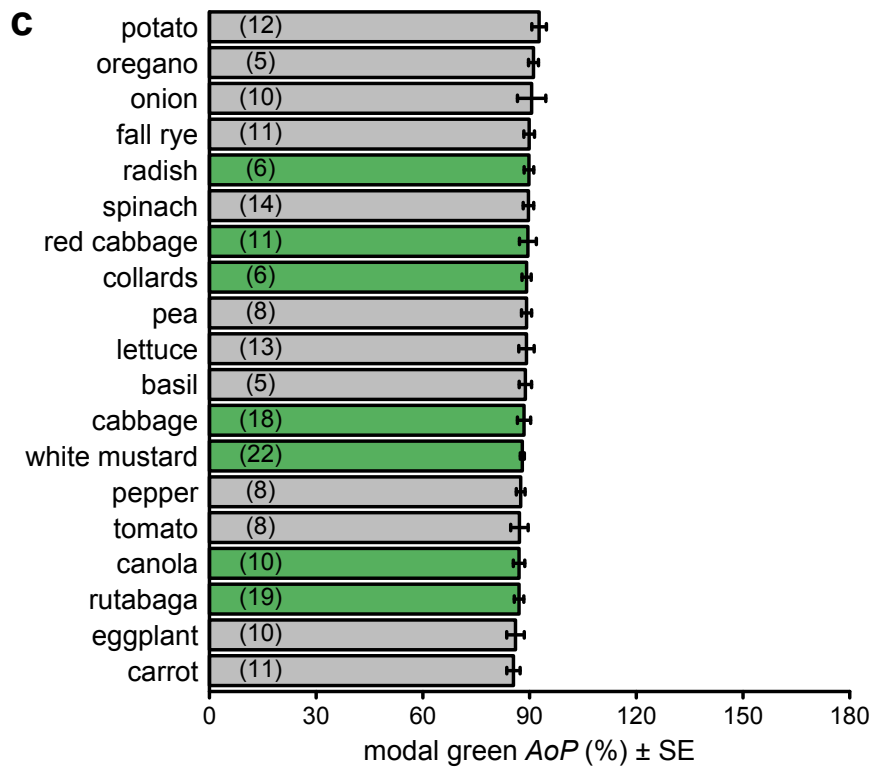

Figure S4. Comparison of intensity (a), degree of linear polarization $(D o L P)(\mathbf{b})$, and axis of polarization $(A o P)(\mathbf{c})$ among host plants (green bars) and non-host plants (grey bars) of Pieris rapae. These measurements used the green color band. Bars show mean or modal values with number of plants measured noted in parentheses in each bar. Bars with different letters differ statistically $(p<0.05)$, as determined by a posthoc Tukey test. 

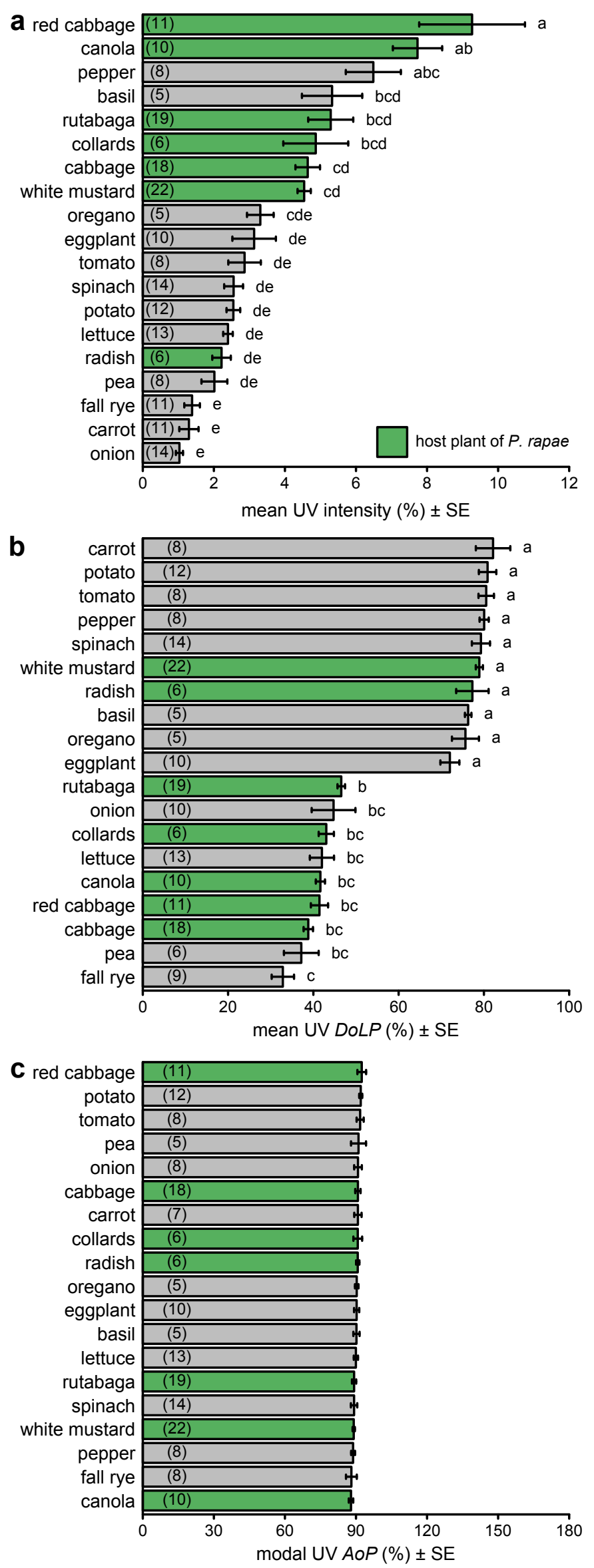

Figure S5. Comparison of intensity (a), degree of linear polarization $(D o L P)(\mathbf{b})$, and axis of polarization $(A o P)(\mathbf{c})$ among host plants (green bars) and non-host plants (grey bars) of Pieris rapae. These measurements used the UV color band. Bars show mean or modal values with number of plants measured noted in parentheses in each bar. Bars with different letters differ statistically $(p<0.05)$, as determined by a posthoc Tukey test. 


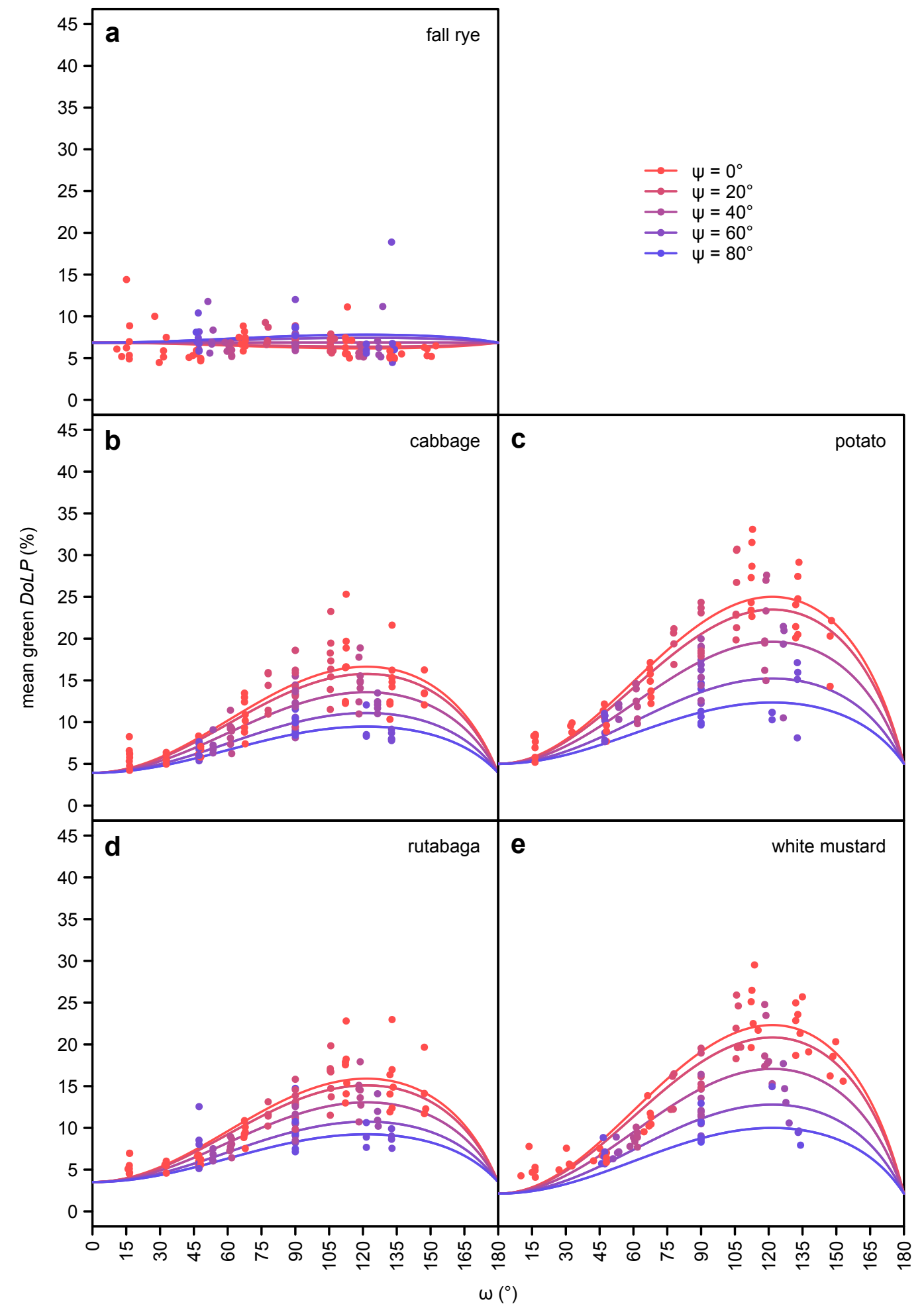

Figure S6. The effect of $\omega$ (angle between observer and light source with the plant at its vertex; see Fig. 1) and $\psi$ (2-dimensional component of $\omega$ perpendicular to the plane passing through both the observer and the plant; see Fig. 1) on the mean degree of linear polarization $(D o L P)$ of the green color band, as measured in five select plant species using photo polarimetry. Cabbage, rutabaga and white mustard are host plants of Pieris rapae. 


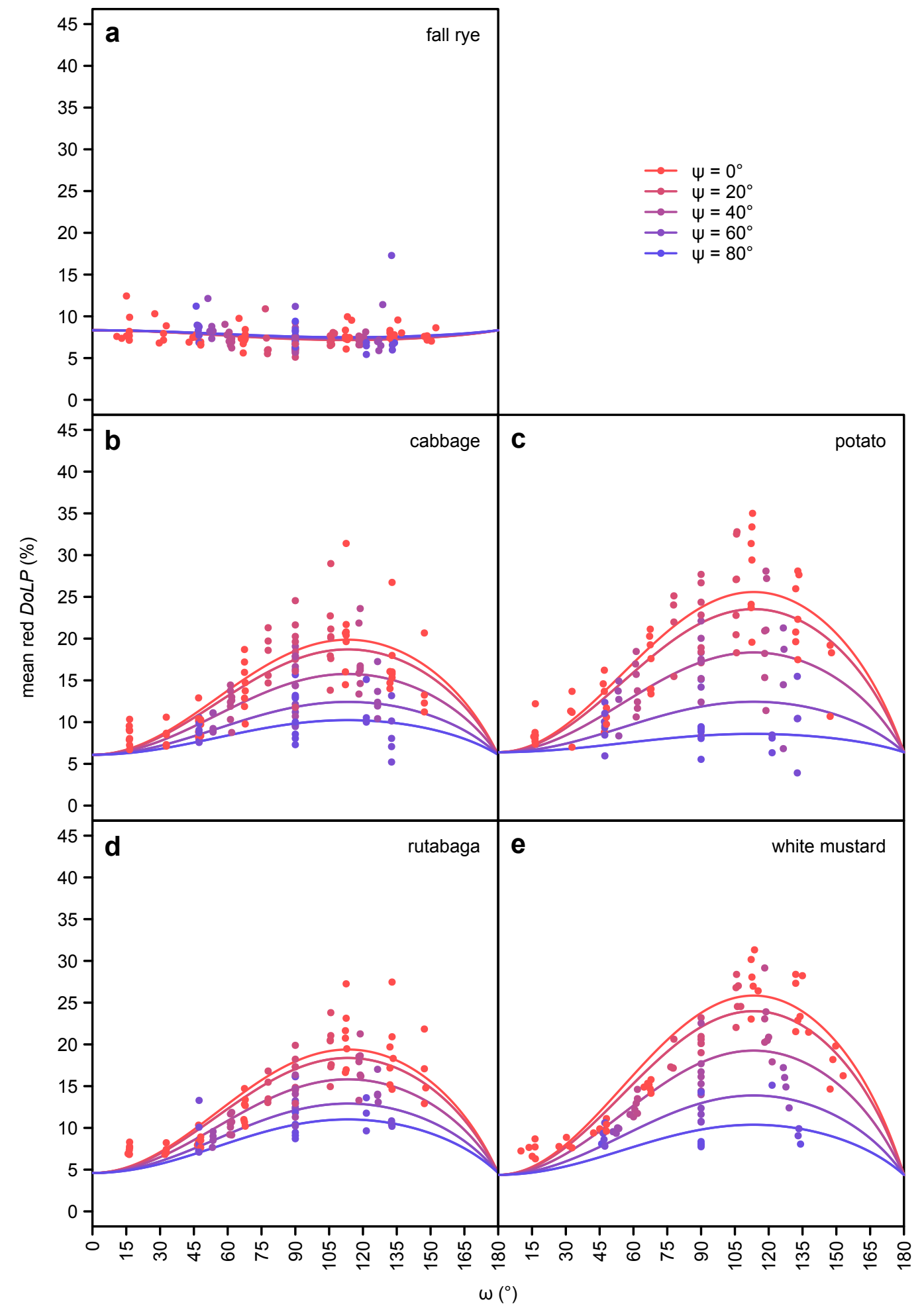

Figure S7. The effect of $\omega$ (angle between observer and light source with the plant at its vertex; see Fig. 1) and $\psi$ (2-dimensional component of $\omega$ perpendicular to the plane passing through both the observer and the plant; see Fig. 1) on the mean degree of linear polarization $(D o L P)$ of the blue color band, as measured in five select plant species using photo polarimetry. Cabbage, rutabaga and white mustard are host plants of Pieris rapae. 


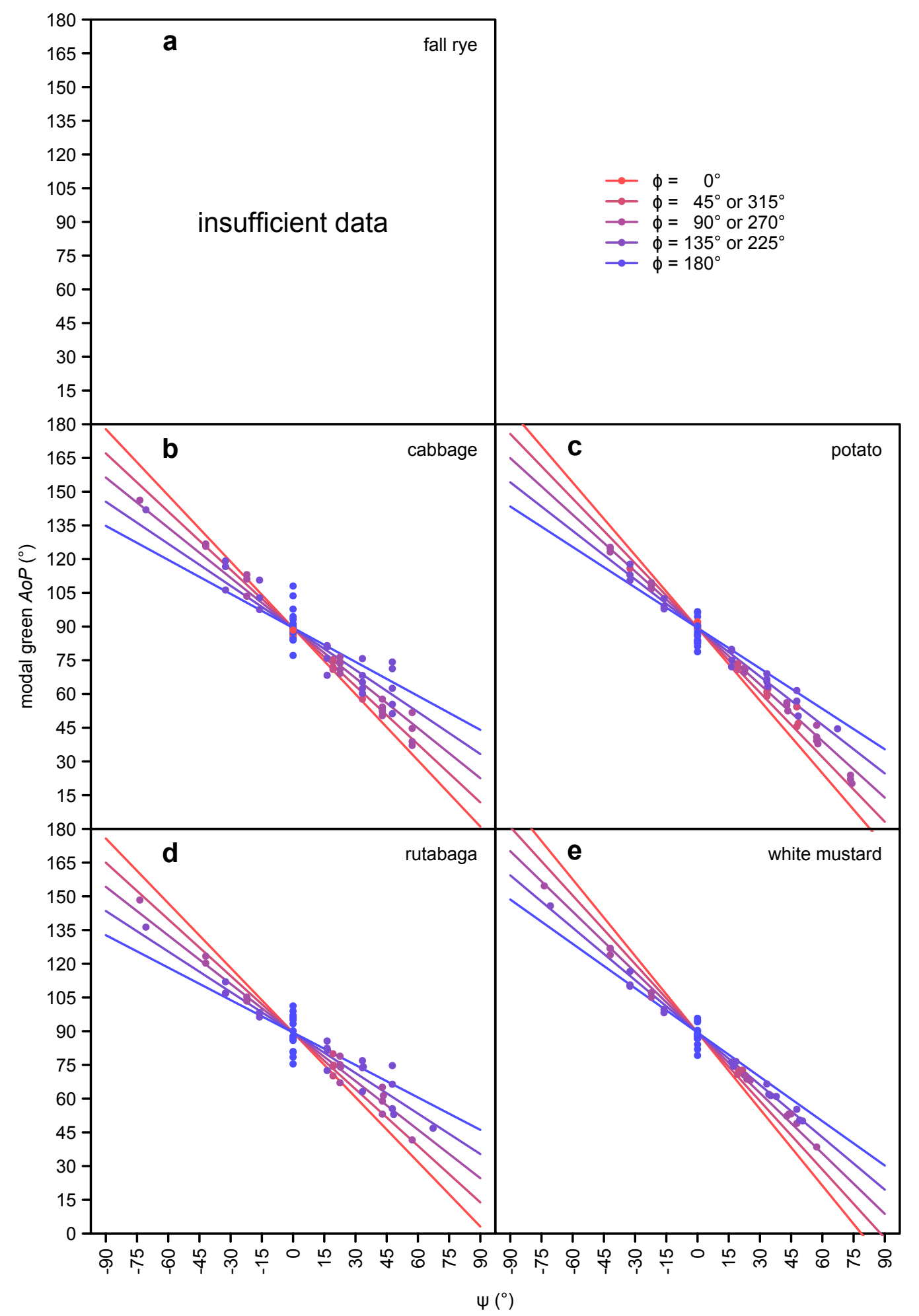

Figure S8. The effect of $\psi$ (2-dimensional component of $\omega$ perpendicular to the plane passing through both the observer and plant; see Fig. 1) and $\phi$ (angle between the azimuth of the observer and the light source; see Fig. 1) on the modal axis of polarization $(A o P)$ of the green color band, as measured in five select plant species using photo polarimetry. Cabbage, rutabaga and white mustard are host plants of Pieris rapae. Fall rye data were excluded from analyses due to an insufficient number of measurements meeting the inclusion criterion $(>10 \%$ of pixels with a degree of linear polarization above $15 \%$ ). 


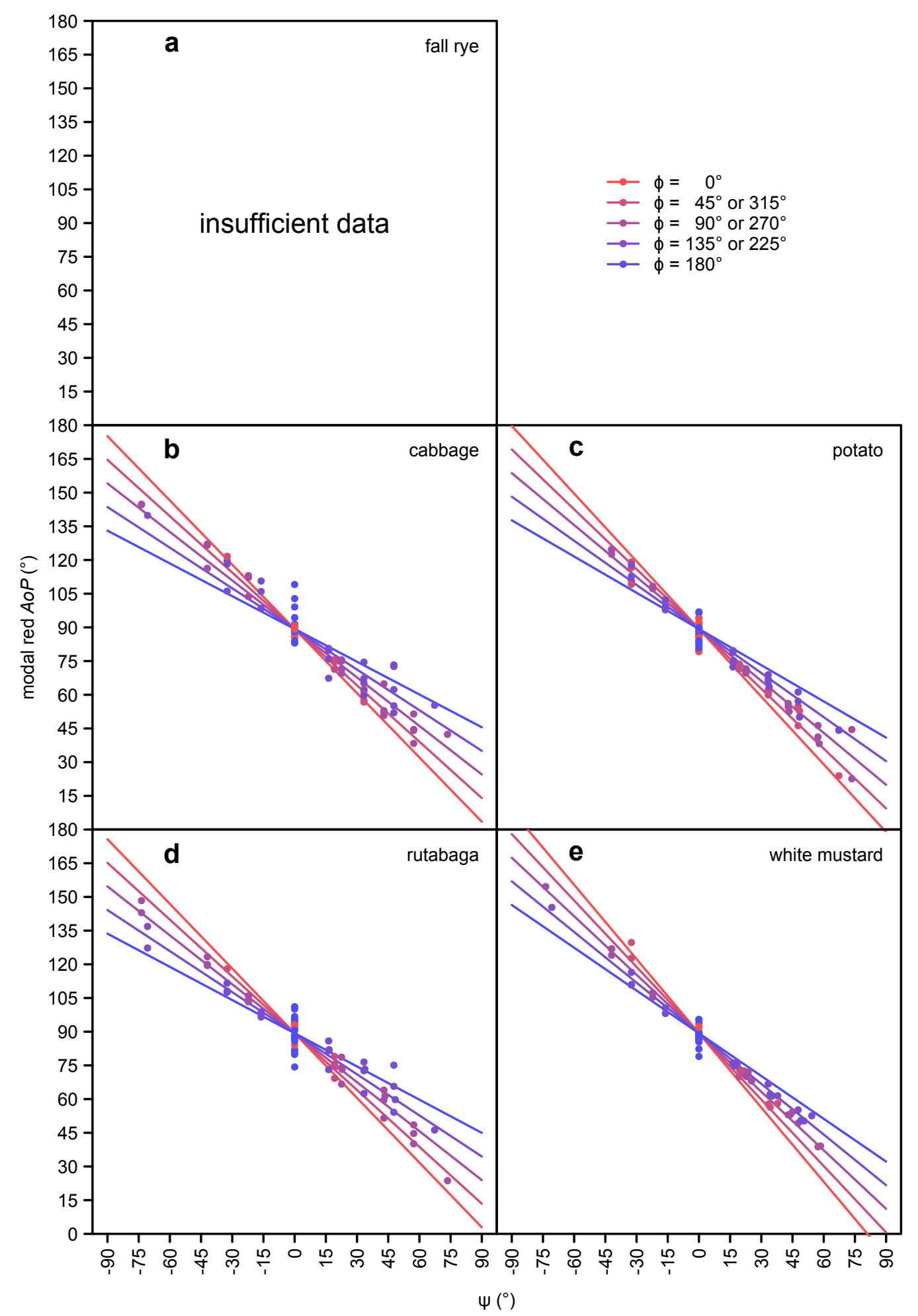

Figure S9. The effect of $\psi$ (2-dimensional component of $\omega$ perpendicular to the plane passing through both the observer and plant; see Fig. 1) and $\phi$ (angle between the azimuth of the observer and the light source; see Fig. 1) on the modal axis of polarization $(A o P)$ of the red color band, as measured in four select plant species using photo polarimetry. Cabbage, rutabaga and white mustard are host plants of Pieris rapae. Fall rye data were excluded from analyses due to an insufficient number of measurements meeting the inclusion criterion $(>10 \%$ of pixels with a degree of linear polarization above $15 \%$ ). 


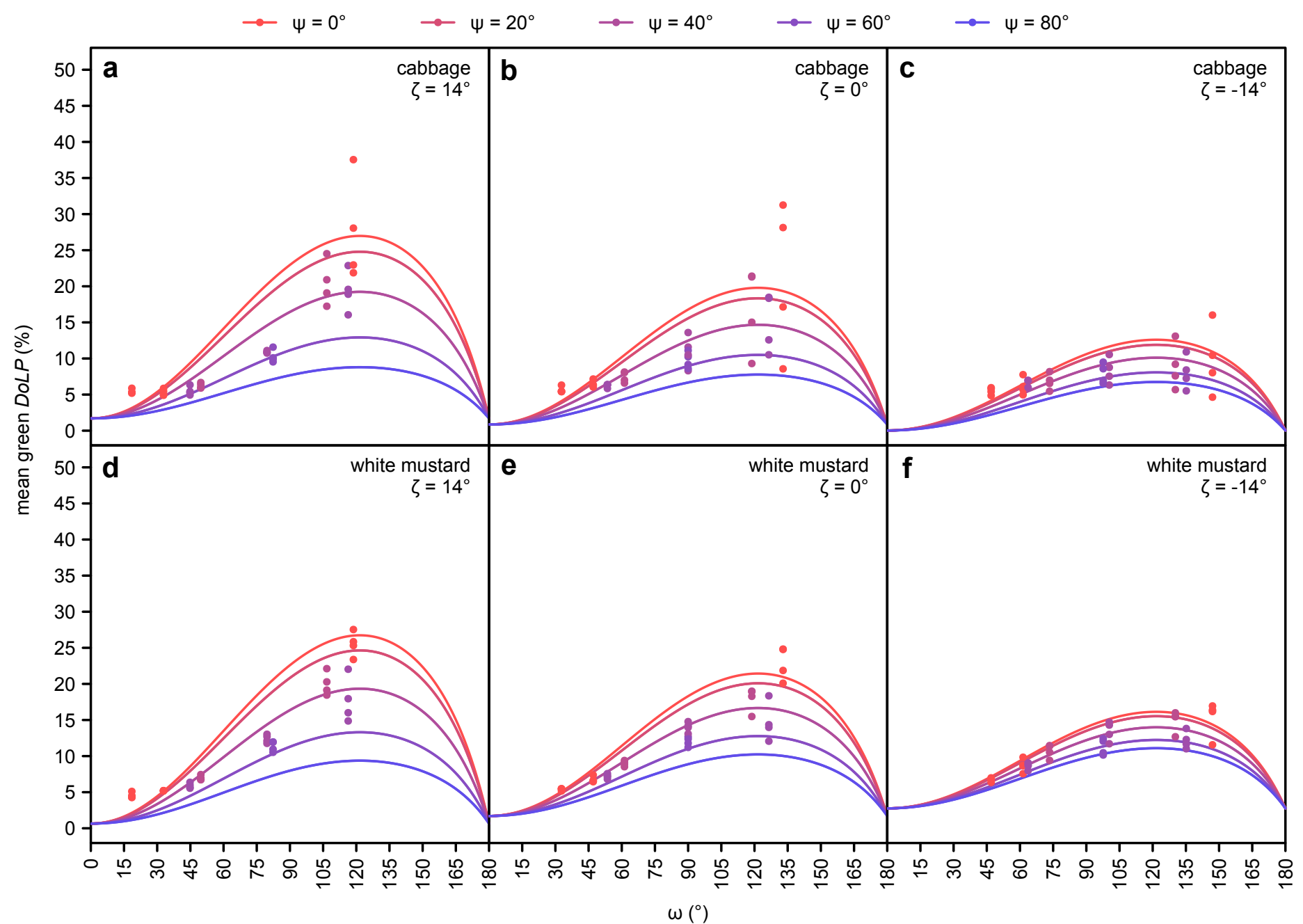

Figure S10. Additional effect of $\zeta$ (elevation of the observer; see Fig. 1) on the mean degree of linear polarization $(D o L P)$ of the green color band, as measured in cabbage and white mustard (host plants of Pieris rapae) using photo polarimetry. 


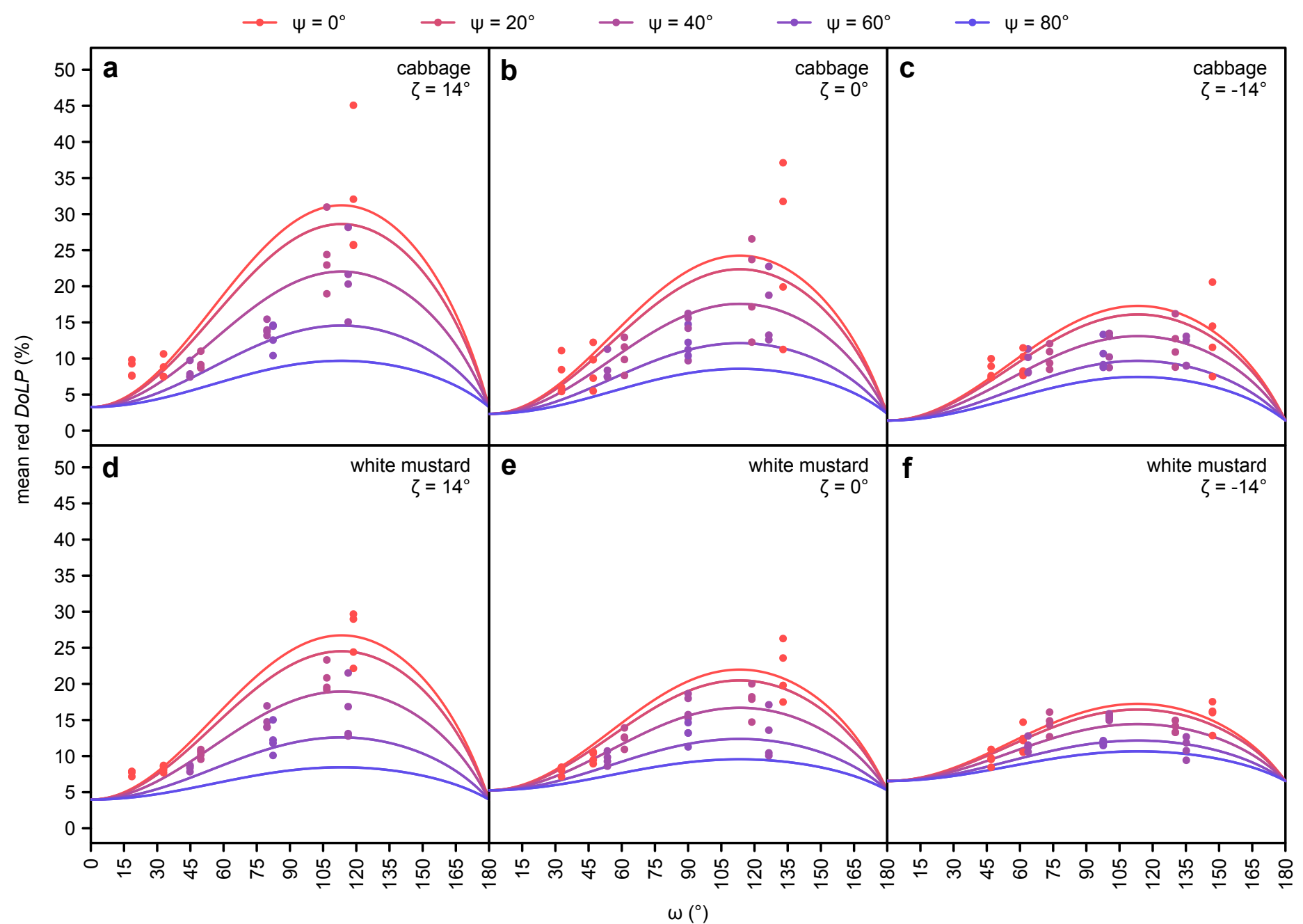

Figure S11. Additional effect of $\zeta$ (elevation of the observer; see Fig. 1) on the mean degree of linear polarization $(D o L P)$ of the red color band, as measured in cabbage and white mustard (host plants of Pieris rapae) using photo polarimetry. 


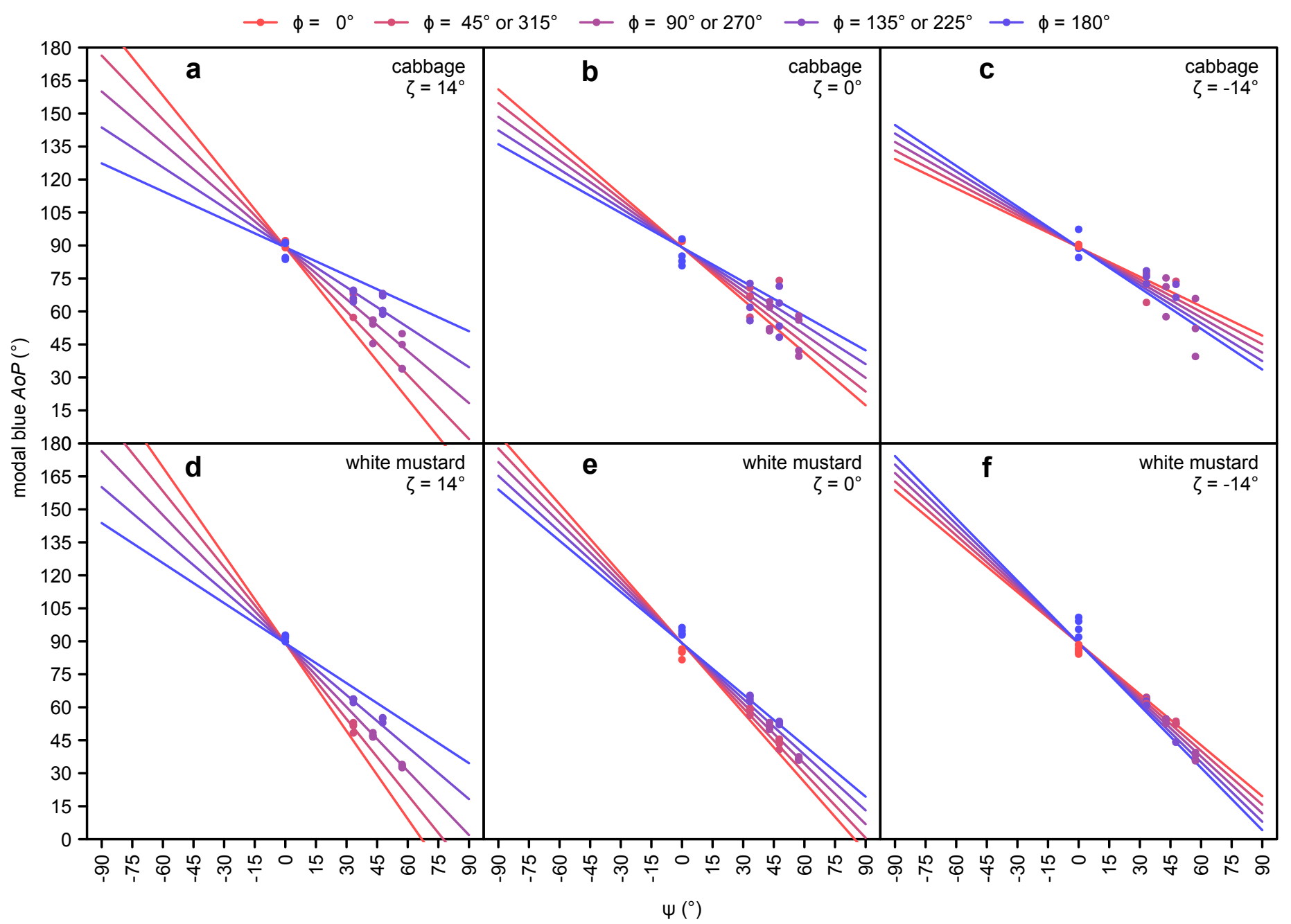

Figure S12. Additional effect of $\zeta$ (elevation of the observer; see Fig. 1) on the modal axis of polarization $(A o P)$ of the blue color band, as measured in cabbage and white mustard (hosts of Pieris rapae) using photo polarimetry. Red and green color band data were excluded from analyses due to an insufficient number of measurements meeting the inclusion criterion $(<10 \%$ of pixels with a degree of linear polarization above $15 \%$ ). 


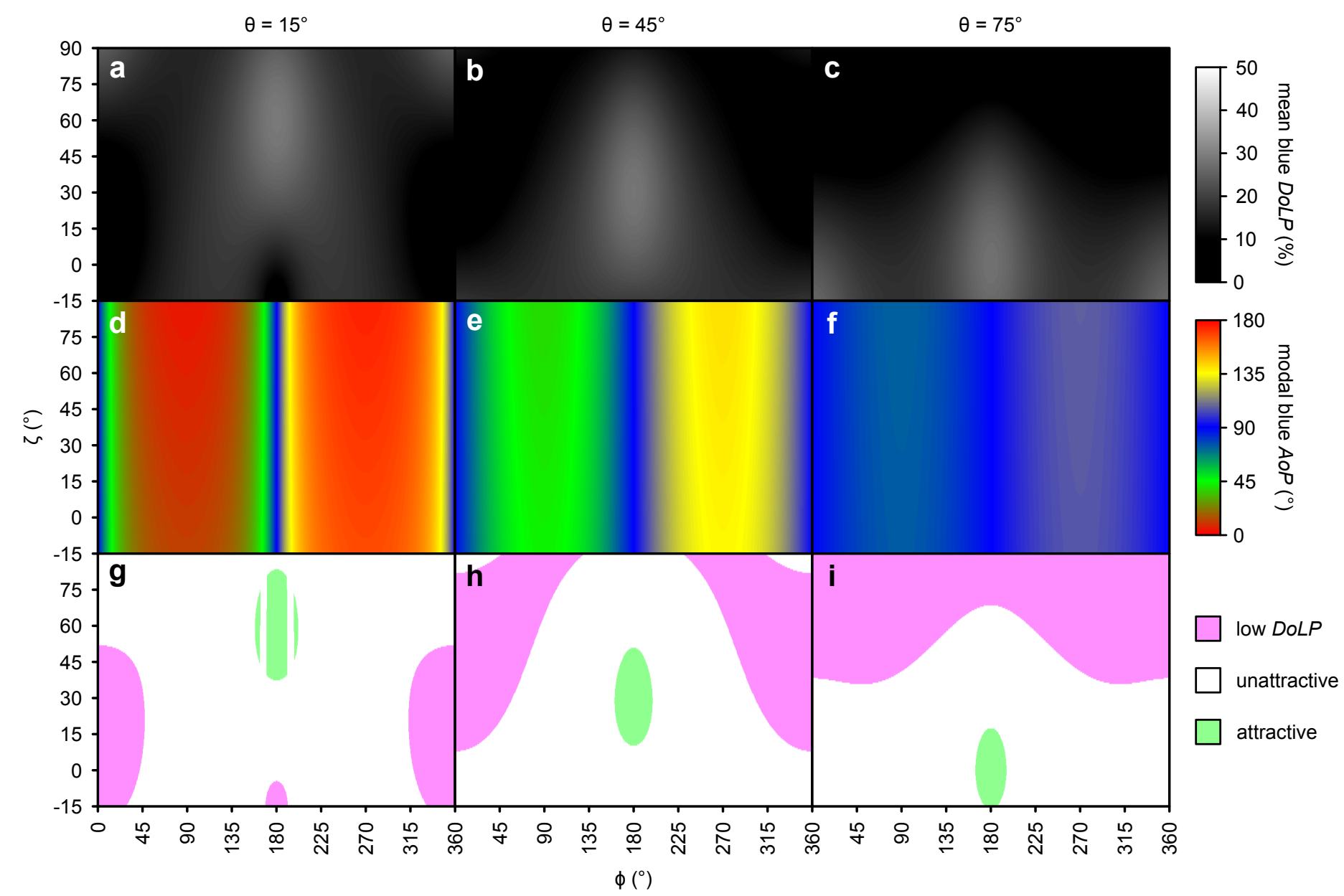

Figure S13. Effects of approach direction (angle between the azimuth of the observer and the light source $(\phi$, see Fig. 1) and elevation of the observer ( $\zeta$; see Fig. 1) on the mean degree of linear polarization $(D o L P)(\mathbf{a}-\mathbf{c})$ and the modal axis of polarization $(A o P)(\mathbf{d}-\mathbf{f})$ of the blue color band of white mustard plants (host of Pieris rapae). Attractiveness of resulting polarization characteristics to Pieris rapae (g-i), based on a previous behavioral study (Blake et al. 2019). Approach trajectories resulting in attractive characteristics $(D o L P=26-36 \%$ and $A o P=0-38,53$ 128 or $\left.143-180^{\circ}\right)$ and unattractive characteristics $\left(D o L P=10-26 \%\right.$ or $\left.A o P=38-53^{\circ}, 128-143^{\circ}\right)$ are shown in green and white, respectively, with pink indicating trajectories resulting in a moderately-attractive low $\operatorname{DoLP}(<10 \%)$. Higher DoLP $(36-60 \%)$ would also be unattractive but were not predicted by these models. These effects changed with light source elevation ( $\theta$; see Fig. 1) which is shown at $15^{\circ}(\mathbf{a}, \mathbf{d}, \mathbf{g}), 45^{\circ}(\mathbf{b}, \mathbf{e}, \mathbf{h})$ and $75^{\circ}(\mathbf{c}, \mathbf{f}, \mathbf{i})$. 